\title{
Thermodynamics as a Consequence of Information Conservation
}

\author{
Manabendra Nath Bera ${ }^{1,2}$, Arnau Riera ${ }^{1,2}$, Maciej Lewenstein ${ }^{1,3}$, Zahra Baghali Khanian ${ }^{1,4}$, and \\ Andreas Winter ${ }^{3,4}$ \\ ${ }^{1}$ ICFO - Institut de Ciencies Fotoniques, The Barcelona Institute of Science and Technology, ES-08860 Castelldefels, Spain \\ ${ }^{2}$ Max-Planck-Institut für Quantenoptik, D-85748 Garching, Germany \\ ${ }^{3}$ ICREA, Pg. Lluis Companys 23, ES-08010 Barcelona, Spain \\ ${ }^{4}$ Departament de Física: Grup d’Informació Quàntica, Universitat Autònoma de Barcelona, ES-08193 Bellaterra (Barcelona), \\ Spain \\ November 30, 2018
}

Thermodynamics and information have intricate interrelations. Often thermodynamics is considered to be the logical premise to justify that information is physical - through Landauer's principle -, thereby also linking information and thermodynamics. This approach towards information has been instrumental to understand thermodynamics of logical and physical processes, both in the classical and quantum domain. In the present work, we formulate thermodynamics as an exclusive consequence of information conservation. The framework can be applied to the most general situations, beyond the traditional assumptions in thermodynamics: we allow systems and thermal baths to be quantum, of arbitrary sizes and even possessing inter-system correlations.

Here, systems and baths are not treated differently, rather both are considered on an equal footing. This leads us to introduce a "temperature"-independent formulation of thermodynamics. We rely on the fact that, for a fixed amount of information, measured by the von Neumann entropy, any system can be transformed to a state with the same entropy that possesses minimal energy. This state, known as a completely passive state, acquires Boltzmann-Gibbs canonical form with an intrinsic temperature. We introduce the notions of bound and free energy and use them to quantify heat and work, respec-

Manabendra Nath Bera: mnbera@gmail.com

Andreas Winter: andreas.winter@uab.cat tively. Guided by the principle of information conservation, we develop universal notions of equilibrium, heat and work, Landauer's principle and universal fundamental laws of thermodynamics. We demonstrate that the maximum efficiency of a quantum engine with a finite bath is in general lower than that of an ideal Carnot engine. We introduce a resource theoretic framework for our intrinsic temperature based thermodynamics, within which we address the problem of work extraction and state transformations. Finally, the framework is extended to multiple conserved quantities.

\section{Introduction}

Thermodynamics constitutes one of the basic foundations of modern science. It not only plays an important role in modern technologies, but offers a basic understanding of a vast range of natural phenomena. Initially, thermodynamics was developed phenomenologically, to address the question of how, and to what extent, heat can be converted into work. Later, with the developments of statistical mechanics, quantum mechanics and relativity, thermodynamics along with its fundamental laws was put on a formal and mathematically rigorous footing [1]. Beyond its original domain of conception, it has been applied in a wide range of contexts. It has been used to describe relativistic phenomena in astrophysics and cosmology, quantum effects in microscopic systems, or very complex systems in biology and chemistry, and even in music [2].

The inter-relation between information and ther- 
modynamics [3] is intricate, and has been studied in the context of Maxwell's demon [4-7], Szilard's engine [8], and Landauer's principle [9-13]. In recent years, classical and quantum information-theoretic approaches helped to understand thermodynamics in the domain of small classical and quantum systems [14-16]. That stimulated a whole new perspective to tackle and extend thermodynamics beyond the standard classical domain. In fact, information theory has recently played an important role in understanding thermodynamics in the presence of inter-system and system-bath correlations [17-19], equilibration processes [20-23], and the foundations of statistical mechanics [24]. One of the most paradigmatic examples of this success is the formulation of quantum thermodynamics within the so-called resource theoretic framework [25], which allows to reproduce standard thermodynamics in the asymptotic limit, when one processes infinitely many copies of the system under consideration. In the finite-copy, or even oneshot limit, the resource theory reveals that the laws of thermodynamics have to be modified to capture finite-size, as well as quantum effects in thermodynamics [26-34].

In the present work, elaborating in the interrelations between information and thermodynamics, we perform an axiomatic construction of thermodynamics and identify the "information conservation" as the crucial underlying property of any theory that respects it. The cornerstone of our construction is the notion of bound energy, which we introduce as the amount of energy locked in a system that cannot be accessed (extracted) by using the set of allowed operations. The bound energy obviously depends on the set of allowed operations: the larger this set is, the smaller the bound energy. We prove that, by taking ( $i$ ) global entropy preserving operations as the set of allowed operations and (ii) infinitely large thermal baths initially uncorrelated with the system, our formalism reproduces standard thermodynamics in its resource theoretic form.

In information theory, the von Neumann entropy is the quantity that measures the amount of information in a system. In this sense, entropy preserving operations are transformations that keep this information constant. All fundamental physical theories, such as classical and quantum mechanics, share the property of conserving information at the basic level. Namely, they have dynamics that is deterministic and bijectively maps the set of possible configurations between any two instants of time. Thus, non- determinism can only appear when some degrees of freedom are ignored, leading to apparent information loss. In classical physics, this information loss is due to deterministic chaos and mixing in nonlinear dynamics. In quantum mechanics, this loss is intrinsic, and occurs due to measurement processes and non-local correlations [35]. Note that the set of entropy preserving operations is larger than the dynamically reversible operations (unitaries) in the sense that they conserve entropy but, unlike unitaries, not the individual probabilities. In other words, we demand only coarse-grained, not microscopic information conservation. In the limit of many copies both coarse-grained and fine-grained information conservation become equivalent (see Sec. 2). While it is known that linear operations that are entropy preserving for all states are unitary [36], it is an open question to what extent coarse-grained information conserving operations can be implemented in the singlecopy setting.

The resource theory of thermodynamics can then be seen as one where condition $(i)$ is sharpened, i.e. an extension of thermodynamics from coarse-grained to fine-grained information conservation, where the operations are global unitaries but still constrained to infinitely large thermal baths with a well defined temperature. Fluctuation theorems can also be considered from this perspective. There, the second law is obtained as a consequence of reversible transformations on initially thermal states or states with a well defined temperature [37-40]. In contrast, the aim of our work is instead to relax condition ( $i i)$, that is, to generalize thermodynamics to be valid for arbitrary environments, irrespective of being thermal, or much larger than the system. This idea is illustrated in the table below.

\begin{tabular}{ccc} 
& Unitaries & $\begin{array}{c}\text { Entropy } \\
\text { preserving } \\
\text { operations } \\
\text { (fine-grained } \\
\text { information } \\
\text { information } \\
\text { conservation) } \\
\text { conservation) }\end{array}$ \\
\hline $\begin{array}{c}\text { Large } \\
\text { thermal } \\
\text { bath }\end{array}$ & $\begin{array}{c}\text { Resource theory of } \\
\text { thermodynamics }\end{array}$ & $\begin{array}{c}\text { Standard } \\
\text { thermodynamics }\end{array}$ \\
\hline $\begin{array}{c}\text { Arbitrary } \\
\text { environment }\end{array}$ & $?$ & Present paper \\
\hline
\end{tabular}

The main obstruction against this generalization lies in the fact that, when allowing for arbitrary states as environment, large amounts of resources 
can be pumped into the system leading to trivial "resource" theories. We are able to circumvent this problem thanks to the notion of bound energy, which intrinsically distinguishes accessible and nonaccessible energy. Our formalism results in a theory in which systems and environments are treated on an equal footing, or in other words, in a "temperature"independent formulation of thermodynamics.

Our work is complementary to other general approaches, where thermodynamics is obtained after inserting some form of thermal state(s) in general mathematical expressions, e.g. [41]. While in these works the mathematical expressions for arbitrary states have no a priori thermodynamic meaning, our construction is built on a physically motivated quantity, the bound energy.

The present temperature-independent thermodynamics is essential in contexts in which the state of the bath can be affected by the system after exchange of heat (see [42] for a review on the notion of temperature). This can be due to the fact of either having a relatively small environment compared to the system, or an environment simply not being thermal. In fact, in current experiments, environments do not have to be necessarily thermal, but can possess quantum coherence or correlations.

The entropy preserving operations make all states with equal energies and entropies thermodynamically equivalent. This allows for representing all the states and thermodynamic processes in a simple energyentropy diagram. We exploit this geometric approach and give a diagrammatic representation for heat, work and other thermodynamic quantities. In this way we are able to reproduce several results of the literature, e.g. the resource theory of thermodynamics applicable for arbitrary quantum systems and environments [43]. Our formalism extends naturally to scenarios with multiple conserved quantities.

The structure of the rest of the paper is as follows: In the following Section 2, we discuss the class of entropy preserving operations, on which our theory is built. Then, in Section 3, we introduce the fundamental notions of bound and free energy, and in Section 4 define the energy-entropy diagram of a system. After that, we develop thermodynamics, from the zeroth law (Section 5) and a discussion of the max-entropy vs. min-energy principle (Section 6), to the first law (Section 7) and the second law of thermodynamics (Section 8). In Section 9 we discuss if, and why not, a third law can be formulated in our setting. Then, in Section 10, we move to the devel- opment of a resource theory based on the previous formalism, which allows us to consider transformation rates between states and visualize the results in the energy-entropy diagram. In Section 11, we outline how the theory so far developed generalizes to the case of multiple conserved quantities. Finally, we summarize our findings in Section 12, and discuss the main obstructions towards an extension of thermodynamics that would be valid both in the single-shot scenario and for non-thermal environments.

\section{Entropy preserving operations, en- tropic equivalence class and intrinsic temperature}

The set of operations that we consider in this framework is the set of, so called, entropy preserving operations. Given a system initially in a state $\rho$, the set of entropy preserving operations are all the operations that arbitrarily change the state, but keep its entropy constant

$$
\rho \rightarrow \sigma \text { s.t. } S(\rho)=S(\sigma),
$$

where $S(\rho):=-\operatorname{Tr} \rho \log \rho$ is the von Neumann entropy. Importantly, an operation that acts on $\rho$ and produces a state with the same entropy, not necessarily preserves entropy when acting on other states. In fact, such entropy preserving operations are in general not linear, since they have to be constrained to some input state. It was shown in Ref. [36] that a quantum channel $\Lambda(\cdot)$ that preserves entropy and, at the same time, respects linearity, i. e. $\Lambda\left(p \rho_{1}+(1-\right.$ p) $\left.\rho_{2}\right)=p \Lambda\left(\rho_{1}\right)+(1-p) \Lambda\left(\rho_{2}\right)$, has to be a unitary.

However, the entropy preserving operations can be microscopically described by global unitaries in the limit of many copies [43]. Given any two states $\rho$ and $\sigma$ with equal entropies $S(\rho)=S(\sigma)$, there exists an additional system of $O(\sqrt{n \log n})$ ancillary qubits and a global unitary $U$ such that, as $n \rightarrow \infty$,

$$
\left\|\operatorname{Tr}_{\text {anc }}\left(U \rho^{\otimes n} \otimes \eta U^{\dagger}\right)-\sigma^{\otimes n}\right\|_{1} \rightarrow 0,
$$

where the partial trace is performed on the ancillary qubits. Here, $\|X\|_{1}:=\operatorname{Tr} \sqrt{X^{\dagger} X}$ is the one-norm. As shown in [43, Thm. 4], the reverse statement is also true. In other words, if two states are related as in Eq. (2), then they also have equal entropies.

It is important to restrict entropy preserving operations that are also energy preserving, as we shall see later. The energy and entropy preserving operations can also be implemented using a global energy 
preserving unitary in the many-copy limit. More explicitly, in [43, Thm. 1], it is shown that having two states $\rho$ and $\sigma$ with equal entropies and energies, i.e. $(S(\rho)=S(\sigma)$ and $E(\rho)=E(\sigma))$, is equivalent to the existence of some energy preserving $U$ and an additional system $A$ with $O(\sqrt{n \log n})$ of ancillary qubits with Hamiltonian $\left\|H_{A}\right\| \leqslant O\left(n^{2 / 3}\right)$ in some state $\eta$, for which (2) is fulfilled. The operator norm $\|X\|$ of a Hermitian operator $X$ is the largest of its eigenvalues in absolute value. Note that the amount of energy and entropy of the ancillary system per copy vanishes in the large $n$ limit.

We expect entropy preserving operations to be also implemented in other ways than taking the limit of many copies. For instance, in Refs. [44, 45], thermal operations are extended to a class of operations, in which a catalyst is allowed to build up correlations with the system. For these operations, the standard Helmholtz free energy singles out as the monotone that establishes the possible transitions between states, in contrast to the case of strict thermal operations, in which all the Rényi $\alpha$-free energies are required. This suggests that entropy preserving operations could also be implemented with a single copy by means of a catalyst that can become correlated with the system. Further investigation in this direction is needed.

Now that the entropy preserving operations have been motivated and introduced, let us classify the set of states of a system in different equivalence classes depending on their entropy. Thereby, we establish a hierarchy of states according to their information content.

Definition 1 (Entropic equivalence class). Two states $\rho$ and $\sigma$ on any quantum system of dimension $d$ are equivalent and belong to the same entropic equivalence class if and only if both have the same von Neumann entropy,

$$
\rho \sim \sigma \quad \text { iff } S(\rho)=S(\sigma) .
$$

We call such two states, $\rho$ and $\sigma$ iso-entropic. Assuming that the system has some fixed Hamiltonian $H$, one can take as a representative element of every class the state that minimizes the energy within it, i.e.,

$$
\gamma(\rho):=\underset{\sigma: S(\sigma)=S(\rho)}{\arg \min } E(\sigma),
$$

where $E(\sigma):=\operatorname{Tr} H \sigma$ is the energy of the state $\sigma$.

The maximum-entropy principle $[46,47]$ identifies the thermal state as the state that maximizes the entropy for a given energy. Conversely, one can show that, for a given entropy, the thermal state also minimizes the energy. We refer to this complementary property as min-energy principle [17, 48, 49]. To be precise, this duality holds for all finite inverse temperatures, $\beta<\infty$, corresponding to energies strictly above the ground state energy and entropies strictly larger then the ground state entropy. Thus, the minenergy principle identifies thermal states as the representative elements of every class, which is

$$
\gamma(\rho)=\frac{\mathrm{e}^{-\beta(\rho) H}}{\operatorname{Tr}\left(\mathrm{e}^{-\beta(\rho) H}\right)} .
$$

The inverse temperature $\beta(\rho)$ is the parameter that labels the equivalence class, to which the state $\rho$ belongs. We denote $\beta(\rho)$ as the intrinsic inverse temperature associated to $\rho$.

The state $\gamma(\rho)$ is often termed the completely passive (CP) state [17]. Note, in this article, we denote the CP states, synonymously, with the $\gamma(H, \beta), \gamma(\rho)$, $\gamma(\beta)$ and $\gamma(T)$, where $H$ is the Hamiltonian of system in the state $\rho$, and $\beta=1 / T$ is the inverse of the temperature $T$. The $\gamma(H, \beta)$ is exclusively used in the cases where the Hamiltonian $H$ of the system is fixed. Moreover, the baths, in thermal equilibrium at certain temperature, are also CP states, and we sometime denote these thermal baths with $\gamma(T)$ and $\gamma(\beta)$.

The completely passive state of the form $\gamma\left(H_{S}, \beta_{S}\right)$ has the following interesting properties $[48,49]$ :

(P1) For a given entropy, it minimizes the energy.

(P2) Energy (entropy) monotonically increases (decrease) with the decrease (increase) in $\beta_{S}$.

(P3) For non-interacting Hamiltonians and identical $\beta_{T}, H_{T}=\sum_{X=1}^{N} \mathbb{I}^{\otimes X-1} \otimes H_{X} \otimes \mathbb{I}^{\otimes N-X}$, the joint complete passive state is the tensor product of the individual ones, i.e., $\gamma\left(H_{T}, \beta_{T}\right)=$ $\otimes_{X=1}^{N} \gamma\left(H_{X}, \beta_{T}\right)$. [48, 49].

\section{Bound and free energies}

Let us now identify two relevant forms of internal energy: the free and the bound energy. The bound energy is motivated as the amount of internal energy that cannot be accessed in the form of work. We emphasize once more that it is a notion that depends on the set of allowed operations. For the set of entropy preserving operations, from which the entropic classes and completely passive states emerge, it is quantified as follows. 
Definition 2 (Bound energy). For a state $\rho$ with the system Hamiltonian $H$, the bound energy in it is

$$
B(\rho):=\min _{\sigma: S(\sigma)=S(\rho)} E(\sigma) .
$$

By the previous discussion, $B(\rho)=E(\gamma(\rho))$, where $\gamma(\rho)$ is the completely passive state, with minimum energy, within the equivalence class to which $\rho$ belongs.

Indeed, $B(\rho)$ is the amount of energy that cannot be extracted further, by exploiting any entropy preserving operations, as guaranteed by the min-energy principle. The above definition of bound energy also has strong connection with information content in the state. It can be easily seen that one could have access to this energy (in the form of work) only, if it allows an outflow of information from the system and vice versa.

In contrast to bound energy, free energy is the part of the internal energy that can be accessed with entropy preserving operations.

Definition 3 (Free energy). For a system $\rho$ with system Hamiltonian $H$, the free energy stored in the system is given by

$$
F(\rho):=E(\rho)-B(\rho),
$$

where $B(\rho)$ is the bound energy in $\rho$.

Note that the free energy as defined in Eq. (7) does not have a preferred temperature, unlike the standard out-of-equilibrium Helmholtz free energy $F_{T}(\rho)$ := $E(\rho)-T S(\rho)$, where the temperature $T$ is decided beforehand with the choice of a thermal bath. Nevertheless, our definition of free energy can be written in terms of both the relative entropy and the out-ofequilibrium free energy as

$$
F(\rho)=T(\rho) D(\rho \| \gamma(\rho))=F_{T(\rho)}(\rho)-F_{T(\rho)}(\gamma(\rho)),
$$

for the intrinsic temperature $T(\rho):=\beta(\rho)^{-1}$ that labels the equivalence class that contains $\rho$. Here the relative entropy is defined as $D(\rho \| \sigma)=$ $\operatorname{Tr}(\rho \log \rho-\rho \log \sigma)$. Let us mention that the standard out-of-equilibrium free energy is also denoted by $F_{\beta}(\rho):=E(\rho)-\beta^{-1} S(\rho)$ in the rest of the manuscript, wherever we find it more convenient.

The notions of bound and free energy, as we consider above, can be extended beyond single systems. If fact, in composite systems they exhibit several interesting properties. For example, they can capture the presence and/or absence of inter-party correlations. To highlight these features, we consider a bipartite system below.

Lemma 4 (Bound and free energy properties). Given a bipartite system with non-interacting Hamiltonian $H_{A} \otimes \mathbb{I}+\mathbb{I} \otimes H_{B}$ in an arbitrary state $\rho_{A B}$ with marginals $\rho_{A / B}:=\operatorname{Tr}_{B / A}\left(\rho_{A B}\right)$. Then, the bound and the free energy satisfy the following properties:

(P4) Bound energy and correlations:

$$
B\left(\rho_{A B}\right) \leq B\left(\rho_{A} \otimes \rho_{B}\right) .
$$

(P5) Bound energy of composite systems:

$$
B\left(\rho_{A} \otimes \rho_{B}\right) \leq B\left(\rho_{A}\right)+B\left(\rho_{B}\right) .
$$

(P6) Free energy and correlations:

$$
F\left(\rho_{A} \otimes \rho_{B}\right) \leq F\left(\rho_{A B}\right) .
$$

(P7) Free energy of composite systems:

$$
F\left(\rho_{A}\right)+F\left(\rho_{B}\right) \leq F\left(\rho_{A} \otimes \rho_{B}\right) .
$$

Ineqs. (9) and (11) are saturated with equality if and only if $A$ and $B$ are uncorrelated, i.e. $\rho_{A B}=$ $\rho_{A} \otimes \rho_{B}$. Ineqs. (10) and (12) become equalities if and only if $\beta\left(\rho_{A}\right)=\beta\left(\rho_{B}\right)$.

Proof. To prove ( $\left.P_{4}\right)$, we have used the fact that, for a fixed Hamiltonian, the bound energy is monotonically increasing with the entropy, i.e.

$$
B(\rho)<B(\sigma) \text { iff } S(\rho)<S(\sigma),
$$

together with the subadditivity property $S\left(\rho_{A B}\right) \leq S\left(\rho_{A} \otimes \rho_{B}\right)$ of the von Neumann entropy.

The proof of $(P 5)$ relies on the definition of the bound energy, where one exploits entropy preserving operations to minimize the energy. Namely, the iso-entropic equivalence relation is stable under tensor product: if $\rho_{A} \sim \sigma_{A}$ and $\rho_{B} \sim \sigma_{B}$, then $\rho_{A} \otimes \rho_{B} \sim \sigma_{A} \otimes \sigma_{B}$, by the additivity of the entropy. This immediately implies Eq. (10). To see that the inequality (10) is saturated iff the states have identical intrinsic temperatures $\beta\left(\rho_{A}\right)=\beta\left(\rho_{B}\right)$, we appeal to Definition 6 and Lemma 7.

The other properties can be easily proven by noting that the total internal energy is sum of local ones: $E\left(\rho_{A B}\right)=E\left(\rho_{A}\right)+E\left(\rho_{B}\right)$, irrespective 
of inter-system correlations. Then, with the definition of free energy $F\left(\rho_{X}\right)=E\left(\rho_{X}\right)-B\left(\rho_{X}\right)$, and properties $\left(P_{4}\right)$ and $(P 5)$, we easily arrive at $(P 6)$ and $\left(P^{r}\right)$.

The above properties allow us to give an additional operational meaning to the free energy $F(\rho)$. Let us recall first that the work that can be extracted from a system in a state $\rho$ when having at our disposal an infinitely large bath at inverse temperature $\beta$ and global entropy preserving operations, is given by

$$
W=F_{\beta}(\rho)-F_{\beta}(\gamma(\beta)),
$$

where $F_{\beta}(\rho)=E(\rho)-\beta^{-1} S(\rho)$ is the standard free energy with respect to the inverse temperature $\beta$ and $\gamma(\beta)$ is the thermal state.

Lemma 5 (Free energy vs. $\beta$-free energy). The free energy $F(\rho)$ corresponds to work extracted by using a bath at the "worst" possible temperature:

$$
F(\rho)=\min _{\beta}\left(F_{\beta}(\rho)-F_{\beta}(\gamma(\beta))\right) .
$$

In words, the free energy of $\rho$ is the minimum extractable work with respect to arbitrary temperature baths. The inverse temperature that achieves the minimum is the inverse intrinsic temperature $\beta(\rho)$.

Proof. It follows from Lemma 14 together with (P7).

\section{Energy-entropy diagram}

Let us describe here the energy-entropy diagram which appears often in the thermodynamics literature and in particular has recently been exploited in Ref. [43]. Given a system described by a Hamiltonian $H$, a state $\rho$ is represented in the energy-entropy diagram by a point with coordinates $x_{\rho}:=(E(\rho), S(\rho))$, as shown in Fig. 1. All physical states reside in a region that is lower bounded by the horizontal axis (i.e. $S=0$ ), corresponding to the pure states, and upper bounded by the convex curve $(E(\beta), S(\beta))$, which represents the thermal states of both positive and negative temperatures. Let us denote such a curve as the thermal boundary. The inverse temperature associated to one point of the thermal boundary is given by the slope of the tangent line in such a point,

$$
\beta=\frac{\mathrm{d} S(\beta)}{\mathrm{d} E(\beta)} .
$$

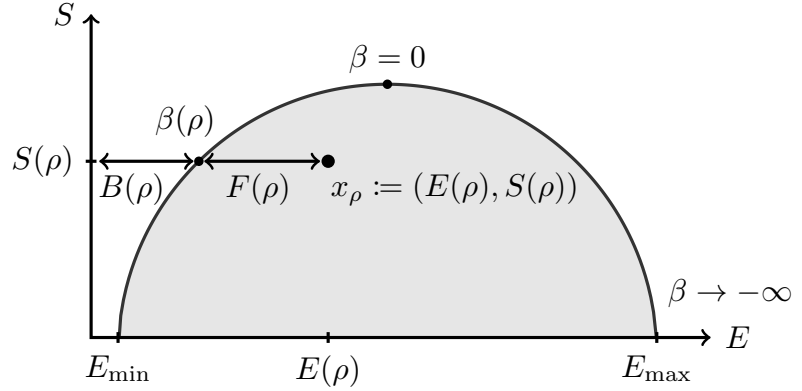

Figure 1: Energy-entropy diagram. Any quantum state $\rho$ is represented in the diagram as a point with coordinates $x_{\rho}:=(E(\rho), S(\rho))$. The free energy $F(\rho)$ is the distance in the horizontal direction from the thermal boundary. The bound energy $B(\rho)$ is the distance in the horizontal direction between the thermal boundary and the energy reference.

In Fig. 1, the free energy and the bound energy are plotted given a state $\rho$. Its free energy $F(\rho)$ can be seen from the diagram as the horizontal distance from the thermal boundary. This is the part of the internal energy which can be extracted without altering system's entropy. The slope of the tangent line of the thermal boundary in that point is the inverse intrinsic temperature $\beta(\rho)$, of the state $\rho$. The bound energy $B(\rho)$ is the distance in the horizontal direction between the thermal boundary and the energy reference and it can in no way be extracted with entropy preserving operations.

Note that in general a point in the energy-entropy diagram represents multiple states, in fact, an entire equivalence class, since different quantum states can have identical entropy and energy. As it is pointed out in [43], the energy-entropy diagram establishes a link between the microscopic and the macroscopic thermodynamics, in the sense that in the macroscopic limit of many copies all the thermodynamic quantities only rely on the energy and entropy per particle. More precisely, all the states with same entropy and energy are thermodynamically equivalent: as was shown in [43], they can be transformed into each other using energy-conserving unitaries in the limit of many copies $n \rightarrow \infty$, and using an ancillary system, which is of sublinear size $O(\sqrt{n \log n})$ and with a Hamiltonian with its Hamiltonian $O\left(n^{2 / 3}\right)$ sublinearly bounded.

\section{Equilibrium and zeroth law}

The zeroth law of thermodynamics establishes the temperature as an intensive quantity, and its the abso- 
lute scaling, in terms of mutual thermal equilibrium. It says that if a system $A$ is in thermal equilibrium with $B$ and again, $B$ is in thermal equilibrium with $C$, then $A$ will also be in thermal equilibrium with $C$. All the systems that are in mutual thermal equilibrium can be classified in a thermodynamical equivalence class, and each class can be labelled with a unique parameter called temperature. In other words, at thermal equilibrium, temperatures of the individual systems will be equal to each other and also to their arbitrary collections, as there would be no spontaneous heat or energy flow in between. When a nonthermal state is brought in contact with a large thermal bath, then the system tends to acquire a thermally equilibrium state with the temperature of the bath. In this equilibration process, the system can exchange both energy and entropy with the bath and thereby minimize its Helmholtz free energy.

However, in the new setup, where a system cannot have access to asymptotically large thermal baths, or in the complete absence of a bath, the equilibration process is expected to be considerably different. In the following, we introduce a formal definition of equilibrium, based on information preservation and intrinsic temperature.

Definition 6 (Equilibrium and zeroth law). Given a collection of systems $A_{1}, \ldots, A_{n}$ with non-interacting Hamiltonians $H_{1}, \ldots, H_{n}$ in a joint state $\rho_{A_{1} \ldots A_{n}}$, we say that they are mutually at equilibrium if and only if they jointly minimize the free energy as defined in (7):

$$
F\left(\rho_{A_{1} \ldots A_{n}}\right)=0 .
$$

Let us consider two systems A and B in the states $\rho_{A}$ and $\rho_{B}$, with corresponding Hamiltonians $H_{A}$ and $H_{B}$, respectively. The equilibrium is achieved when they jointly attain an iso-entropic state with minimum energy. The corresponding equilibrium state is then a completely passive state $\gamma\left(H_{A B}, \beta_{A B}\right)$ with the joint Hamiltonian $H_{A B}=H_{A} \otimes \mathbb{I}+\mathbb{I} \otimes H_{B}$. Further, the joint completely passive state, following (P3), is

$$
\gamma\left(H_{A B}, \beta_{A B}\right)=\gamma\left(H_{A}, \beta_{A B}\right) \otimes \gamma\left(H_{B}, \beta_{A B}\right),
$$

where the local systems assume their respective completely passive states, importantly with the same $\beta_{A B}$. Recall that $\gamma\left(H_{X}, \beta_{Y}\right)=$ $e^{-\beta_{Y} H_{X}} / \operatorname{Tr}\left(e^{-\beta_{Y} H_{X}}\right)$. Note, for interaction systems, i.e. $H_{A B} \neq H_{A} \otimes \mathbb{I}+\mathbb{I} \otimes H_{B}$, one could minimize global energy using global entropy preserving operation too. This minimum energy state will give rise to a global equilibrium (completely passive ) state with intrinsic global temperature. However, the reduced state, after tracing out one sub-system, may not be a completely passive state.

Another consequence of the definition is that if two arbitrary completely passive states $\gamma\left(H_{A}, \beta_{A}\right)$ and $\gamma\left(H_{B}, \beta_{B}\right)$ have $\beta_{A} \neq \beta_{B}$, then their combined equilibrium state $\gamma\left(H_{A B}, \beta_{A B}\right)=\gamma\left(H_{A}, \beta_{A B}\right) \otimes$ $\gamma\left(H_{B}, \beta_{A B}\right)$ can still have a smaller bound energy without altering the total information content. Therefore, the associated joint completely passive state acquires a unique $\beta_{A B}$ such that

$E\left(\gamma\left(H_{A}, \beta_{A}\right)\right)+E\left(\gamma\left(H_{B}, \beta_{B}\right)\right)>E\left(\gamma\left(H_{A B}, \beta_{A B}\right)\right)$,

which is follows immediately from the min-energy principle and (P5). Moreover, if $\beta_{A} \geq \beta_{B}$, then Eq. (19) dictates a bound on $\beta_{A B}$, as expressed in the following lemma.

Lemma 7. Any iso-entropic equilibration process between $\gamma\left(H_{A}, \beta_{A}\right)$ and $\gamma\left(H_{B}, \beta_{B}\right)$, with $\beta_{A} \geq \beta_{B}$, leads to a state $\gamma\left(H_{A B}, \beta_{A B}\right)$ of mutual equilibrium, where $\beta_{A B}$ satisfies

$$
\beta_{A} \geq \beta_{A B} \geq \beta_{B} .
$$

Proof. For $\beta_{A}=\beta_{B}$, (P3) and (P5) immediately lead to $\beta_{A}=\beta_{A B}=\beta_{B}$. What we want to show is that, for $\beta_{A}>\beta_{B}$, the equilibration leads to $\beta_{A}>\beta_{A B}>\beta_{B}$. This can be seen from the information preservation condition on the equilibration process. Assume the contrary, say that after the equilibration the final inverse temperature is $\beta_{A B} \geq \beta_{A}>\beta_{B}$. But according to (P2), the entropy is monotonically decreasing with $\beta$, hence we would conclude that the total entropy of the system has decreased, contradiction to the assumption of an iso-entropic process. Likewise, $\beta_{A}>\beta_{B} \geq \beta_{A B}$ leads to the contradiction that the total entropy of the system would have increased. Therefore, the only possibility remaining is $\beta_{A}>\beta_{A B}>\beta_{B}$.

Now, with a clear notion of equilibration and equilibrium state, which has minimum internal energy for a fixed information content, we can restate the zeroth law in terms of the intrinsic temperature. By definition, the global completely passive state has minimum internal energy and one cannot extract free energy by using global entropy preserving operations. Further, a global completely passive state not only 
assures that the individual states are also completely passive but also enforces that they share the same intrinsic temperature $\beta$ and has vanishing inter-system correlations.

We can recover the traditional notion of thermal equilibrium as well as zeroth law. In traditional thermodynamics, the thermal bath is considerably much larger than the system under consideration, and is initially in a completely passive state with a predefined temperature. With respect to the bath Hamiltonian $H_{B}$, it can be expressed as $\gamma_{B}=$ $e^{-\beta_{B} H_{B}} / \operatorname{Tr}\left(e^{-\beta_{B} H_{B}}\right)$. Now, if the state $\rho_{S}$ of the system $S$ (with $|S| \ll|B|$ ) with Hamiltonian $H_{S}$ is brought in contact with the thermal bath, then the global thermal equilibrium state will be a completely passive state, i.e. $\gamma_{B} \otimes \rho_{S} \stackrel{\Lambda^{e p}}{\longrightarrow} \gamma_{B}^{\prime} \otimes \gamma_{S}^{\prime}$, with a global inverse equilibrium temperature $\beta_{e}$. With $|B| \gg|S|$, one now easily sees that $\beta_{e} \approx \beta_{B}$ and $\gamma_{S}^{\prime} \approx \gamma\left(H_{S}, \beta_{B}\right)=e^{-\beta_{B} H_{S}} / \operatorname{Tr}\left(e^{-\beta_{B} H_{S}}\right)$.

\section{Max-entropy principle}

\section{vs. min-energy principle}

Let us mention that our framework, which is based on the principle of information conservation, is suited for the work extraction problem. It assumes that the system has mechanisms to release energy to some battery or classical field, but it cannot interchange entropy with it. This contrasts with other situations in spontaneous equilibration, in which the system is assumed to evolve keeping constant the conserved quantities. In such scenario, the equilibrium state is described by the principle of maximum entropy, that is, the state the maximizes the entropy given the conserved quantities.

In the context of the maximum entropy principle, let us introduce the absolute athermality as (cf. [43]):

Definition 8 (Athermality). The athermality of a system in a state $\rho$ and Hamiltonian $H$ is the amount of entropy (information) that the system can still accommodate without increasing its energy, i.e.

$$
A(\rho):=\max _{\sigma: E(\sigma)=E(\rho)} S(\sigma)-S(\rho),
$$

where the maximization is over all states $\sigma$ with energy $E(\rho)$.

The state $\sigma$ that maximizes (21) is obviously a thermal state. In particular, it is the equilibrium state of an equilibration process guided by the maximum entropy principle, where the energy of the system remains constant. Let us call the temperature of such thermal state the spontaneous equilibration temperature, and denote it by $\tilde{\beta}(\rho)$. The athermality corresponds to the amount of entropy produced during such an equilibration process.

Note that the spontaneous equilibration temperature $\tilde{\beta}(\rho)$ always differs from the intrinsic temperature $\beta(\rho)$ unless $\rho$ is thermal. In other words, the maximum entropy and minimum energy principles lead to different equilibrium temperatures. As both entropy and energy are monotonically increasing functions of temperature, the equilibrium temperature determined by the minimum energy principle is always smaller than the one determined by the maximum entropy principle. This is represented in the energy entropy diagram of Fig. 2, where the athermality can be seen as the vertical distance from a state $\rho$ to the thermal boundary. The point in which the thermal boundary intersects with the vertical line $E=E(\rho)$ represents the equilibrium thermal state given by the max-entropy principle. Its temperature $\tilde{\beta}(\rho)$ corresponds to the slope of the tangent line of the thermal boundary at that point.

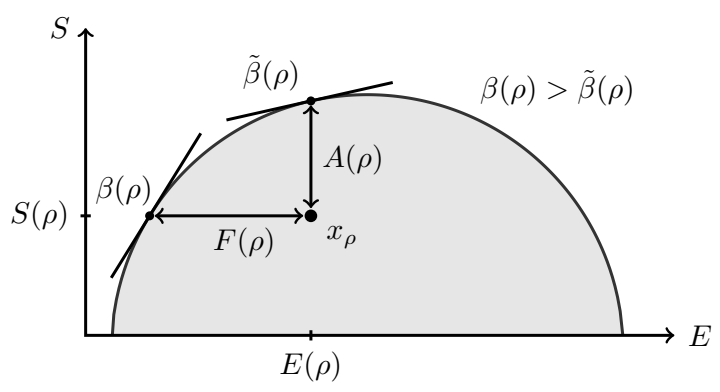

Figure 2: Representation of the free energy $F(\rho)$ and the athermality $A(\rho)$ of a state $\rho$ in the energy-entropy diagram. The intrinsic temperature $\beta(\rho)$ and the spontaneous equilibration temperature $\tilde{\beta}(\rho)$ satisfy $\beta(\rho)>$ $\tilde{\beta}(\rho)$ due to the concavity of the thermal boundary.

We have chosen the name "athermality" for the quantity defined in (8) in agreement with Ref. [43]. There, a quantity called $\beta$-athermality is defined by

$$
A_{\beta}(\rho):=\beta E(\rho)-S(\rho)+\log Z_{\beta},
$$

with $Z_{\beta}=\operatorname{Tr}\left(\mathrm{e}^{-\beta H}\right)$ the partition function. It is introduced to characterise the energy-entropy diagram as the set of all points with positive entropy $S(\rho) \geq 0$ and positive $\beta$-athermality $A_{\beta}(\rho) \geq 0$ for all $\beta \in \mathbb{R}$.

Lemma 9 (Athermality vs. $\beta$-athermality). The athermality of a state $\rho$ can be written in terms 
of the $\beta$-athermalities as

$$
A(\rho)=\min _{\beta} A_{\beta}(\rho),
$$

where the minimum is attained by the spontaneous equilibration temperature $\tilde{\beta}(\rho)$.

Proof. We prove the lemma by giving a geometric interpretation to the $\beta$-athermality in the energyentropy diagram. In Fig. 3, let us consider the tangent line to the thermal boundary with slope $\beta$. Such a straight line is formed by the set of all points satisfying

$$
S-S_{\beta}=\beta\left(E-E_{\beta}\right),
$$

where $S_{\beta}$ and $E_{\beta}$ are respectively the entropy and the energy of the point on the thermal boundary that lies on the line. The vertical distance (difference in entropy) of this line from a point with coordinates $(E(\rho), S(\rho))$ reads

$$
\begin{aligned}
S_{*}-S(\rho) & =\beta\left(E(\rho)-E_{\beta}\right)-S(\rho) \\
& =\beta E(\rho)-S(\rho)-\beta\left(E_{\beta}-T S_{\beta}\right),
\end{aligned}
$$

where $S_{*}$ is the entropy of the line at $E=E(\rho)$ and $T=\beta^{-1}$. By noticing that $\log Z_{\beta}=\beta\left(E_{\beta}-\right.$ $\left.T S_{\beta}\right)$, we get that the $\beta$-athermality $A_{\beta}(\rho)$ of $\rho$ is the vertical distance of $x_{\rho}$ from the line with slope $\beta$ tangent to the thermal boundary. Eq. (23) trivially follows from this.

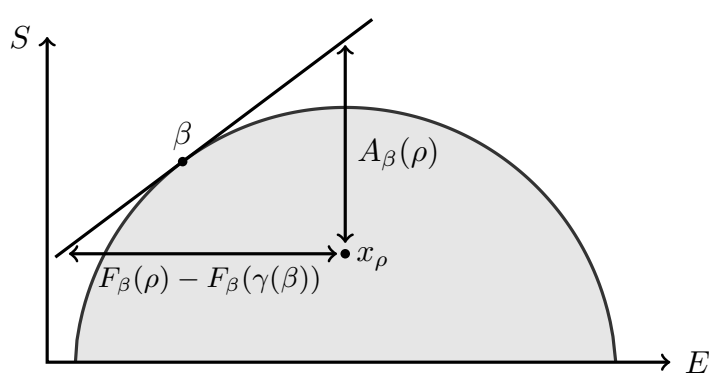

Figure 3: Representation of the $\beta$-free energy difference $F_{\beta}(\rho)-F_{\beta}(\gamma(\beta))$ and the $\beta$-athermality $A_{\beta}(\rho)$ for a state $\rho$ with coordinates $x_{\rho}$ in the energy-entropy diagram.

By identifying the $\beta$-free energy in Eq. (22), the $\beta$-athermality can be written as

$$
A_{\beta}(\rho)=\beta\left(F_{\beta}(\rho)-F_{\beta}(\gamma(\beta))\right),
$$

that is, it is $\beta$ times the work that can be potentially extracted from a state $\rho$ when having an infinite bath uncorrelated from the system at temperature $\beta$ (see Eq. (14)). From Eq. (26), we see that the difference in $\beta$-free energies $F_{\beta}(\rho)-F_{\beta}(\gamma(\beta))$ can be represented in the energy-entropy diagram as the horizontal distance from the point $x_{\rho}$ to the tangent line with slope $\beta$. This is represented in Fig. 3. Hence, given an infinitely large bath at some inverse temperature $\beta$, all the states with the same work potential lie on a line with slope $\beta$ in the energy-entropy diagram. The work extracted from a state $\rho$ can be decomposed into its free energy $F(\rho)$, the part of energy that could have been extracted without bath, and the rest, i.e. the part of the energy that has been accessed thanks to the bath. This latter part is closely related to heat. The definitions of work and heat are discussed in the next section.

Furthermore, thanks to Lemmas 5 and 9, both the free energy and the athermality can be expressed in terms of a minimization over standard $\beta$-free energies

$$
\begin{aligned}
& F(\rho)=\min _{\beta}\left(F_{\beta}(\rho)-F_{\beta}(\gamma(\beta))\right), \\
& A(\rho)=\min _{\beta}\left(\beta\left(F_{\beta}(\rho)-F_{\beta}(\gamma(\beta))\right)\right),
\end{aligned}
$$

where the minimum is attained by the intrinsic temperature $\beta(\rho)$ and the spontaneous equilibration temperature $\tilde{\beta}(\rho)$, respectively.

Note that, while for positive temperatures both the athermality and the free energy are a measure of out of equilibrium, for negative temperatures, states with small athermality are highly active and have huge free energies.

Let us finally show that in the equilibration of a hot body in contact with a cold one, the intrinsic temperature and the spontaneous equilibration one are not so much different. In Fig. 4, we sketch the energy-entropy diagram for the particular case of the equilibration of a system composed of two identical subsystems initially at different temperatures $\beta_{A}$ and $\beta_{B}$. As expected and due to the concavity of the thermal boundary, the equilibrium temperature $\beta_{E}^{-1}$ given by the constant energy constraint (max-entropy principle) is larger that the one given by the constant entropy constrain (min-energy principle), $\beta_{S}^{-1}$, i.e. $\beta_{E}<\beta_{S}$. The difference in bound energies of these two thermal states corresponds precisely to the work extracted in the constant entropy scenario.

\section{Work, heat and the first law}

In thermodynamics, the first law deals with the conservation of energy. In addition, it dictates the distri- 


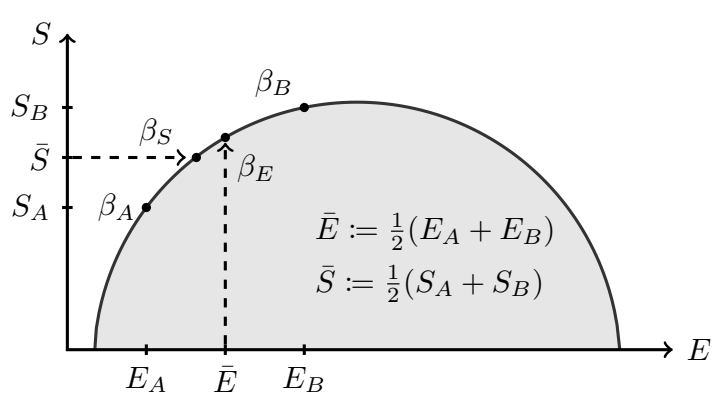

Figure 4: Energy-entropy diagram of a system with Hamiltonian $H$. Two systems with the same Hamiltonian $H$ and initially at different temperatures $\beta_{A}$ and $\beta_{B}$ equilibrate to different temperature depending on the approach taken: minimum energy principle vs. maximum entropy principle. The equilibrium temperature when entropy is conserved $\beta_{S}^{-1}$ is always smaller than the equilibrium temperature when energy is conserved $\beta_{E}^{-1}$, i. e. $\beta_{S}>\beta_{E}$.

bution of energy over work and heat, that are the two forms of thermodynamically relevant energy.

Let us define a thermodynamic process involving a system $A$ and a bath $B$ as a transformation $\rho_{A B} \rightarrow$ $\rho_{A B}^{\prime}$ that conserves the global entropy $S\left(\rho_{A B}\right)=$ $S\left(\rho_{A B}^{\prime}\right)$. In standard thermodynamics, the bath is assumed to be initially in a thermal state and completely uncorrelated from the system. In such a scenario, the heat dissipated in the process is usually defined as the change in the internal energy of the bath, $\Delta Q=E\left(\rho_{B}^{\prime}\right)-E\left(\rho_{B}\right)$, where $\rho_{B}^{(\prime)}=\operatorname{Tr}_{A} \rho_{A B}^{(\prime)}$ is the reduced state of the bath. Here, and in the following, primed quantities refer to the state after the process, whereas their unprimed versions refer to the state before the process. This definition, however, may have some limitations and alternative definitions have been discussed recently [19]. In particular the information theoretic approach from [19] suggests that heat be defined as $\Delta Q=T_{B} \Delta S_{B}$ with $T_{B}$ being the temperature and $\Delta S_{B}=S\left(\rho_{B}^{\prime}\right)-S\left(\rho_{B}\right)$ the change in von Neumann entropy of the bath. Note that $\Delta S_{B}=-\Delta S(A \mid B)$ is also the conditional entropy change in system $A$, conditioned on the bath $B$, where $S(A \mid B)=S\left(\rho_{A B}\right)-S\left(\rho_{B}\right)$, which can be understood as the information flow from the system to the bath in the presence of correlations.

In the present approach, we go beyond the restriction that the environment has to have a definite predefined temperature, and be in a state of the BoltzmannGibbs form. For an arbitrary environment, which could even be athermal, we propose to quantify the heat transfer in terms of bound energies, as follows.

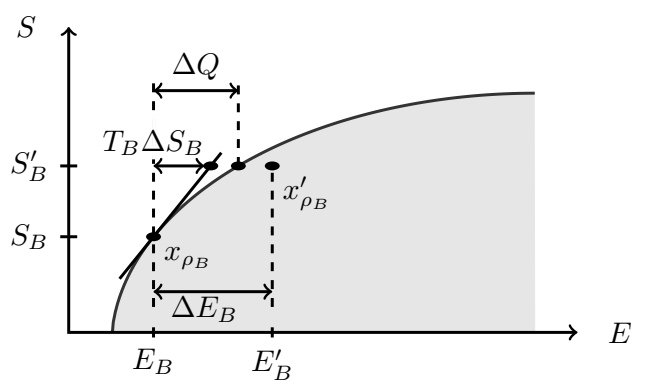

Figure 5: Representation in the energy-entropy diagram of the different notions of heat: $\Delta Q$ as change of the bound energy of the bath, $\Delta E_{B}$ as the change in internal energy, and $T_{B} \Delta S_{B}$. In this example, the bath is initially thermal but this does not have to be necessarily the case.

Definition 10 (Heat). Given a system $A$ and its environment $B$, the heat dissipated by the system $A$ in the process $\rho_{A B} \stackrel{\Lambda^{e p}}{\longrightarrow} \rho_{A B}^{\prime}$ is defined as the change in the bound energy of the environment $B$, i.e.

$$
\Delta Q:=B\left(\rho_{B}^{\prime}\right)-B\left(\rho_{B}\right),
$$

where $B\left(\rho_{B}\right)$ and $B\left(\rho_{B}^{(\prime)}\right)$ are the initial and final bound energy of the bath $B$, respectively.

Note that heat is a somewhat process dependent quantity from the point of view of the work system $A$, in the sense that there can be different processes with the same initial and final marginal state for $A$, but different marginals for $B$. Because the global process is entropy preserving, processes that lead to the same marginal for $A$, but different marginals with different entropies for $B$, necessarily imply a different amount of correlations between $A$ and $B$, as measured by the mutual information $I(A: B):=S_{A}+S_{B}-S_{A B}$. Namely, $\Delta S_{A}+\Delta S_{B}=\Delta I(A: B)$.

Lemma 11 (Connections among definitions of heat). Given a system $A$ and its environment $B$, the heat disspated by $A$ in a globally entropy preserving process $\rho_{A B} \stackrel{\Lambda^{e p}}{\longrightarrow} \rho_{A B}^{\prime}$ can be written as

$$
\Delta Q=\int_{S_{B}}^{S_{B}^{\prime}} T(s) d s,
$$

where $S_{B}^{(\prime)}=S\left(\rho_{B}^{(\prime)}\right)$ is the initial (final) entropy of $B$, and $T(S)$ is the intrinsic temperature of the thermal state of $B$ with entropy $S$. Because of the continuity of $T(S)$, there exists hence an $s_{*} \in\left[S_{B}, S_{B}^{\prime}\right]$ such that

$$
\Delta Q=T\left(s_{*}\right) \Delta S_{B} .
$$

Thus, Definition 10 becomes $\Delta Q=T \Delta S_{B}$ in the limit of small entropy changes along the process, 
in which case $T\left(S_{B}\right) \approx T\left(S_{B}^{\prime}\right) \approx T\left(s_{*}\right)$, while it differs from the common definition in general, and $\Delta Q \neq \Delta E_{B}$.

In the case that the initial state of the environment is thermal $\rho_{B}=\gamma\left(H_{B}, T^{-1}\right)=$ $\mathrm{e}^{-H_{B} / T} / \operatorname{Tr}\left(\mathrm{e}^{-H_{B} / T}\right)$ with Hamiltonian $H_{B}$ at temperature $T$, the heat is lower and upper bounded by

$$
T \Delta S_{B} \leq \Delta Q \leq \Delta E_{B},
$$

where the three quantities have been represented in the energy-entropy diagram in Fig. 5. If, in addition, the process perturbs the environment only slightly, $\rho_{B}^{\prime}=\rho_{B}+\delta \rho_{B}$, then the three definitions of heat coincide, in the sense that

$$
T \Delta S_{B}+O\left(\delta \rho_{B}^{2}\right)=\Delta Q=\Delta E_{B}-O\left(\delta \rho_{B}^{2}\right) .
$$

That is, in the limit of large thermal baths, the three definitions become equivalent.

Proof. Eq. (29) is a consequence of the definitions of heat (28) and bound energy (6), together with Eq. (5). Next, Eq. (30) is proven by using the mean value theorem for the function $T(s)$. The lower bound in (31) is due to the concavity of the thermal boundary together with the reminder theorem of a first order Taylor expansion of the thermal boundary (see Fig. 5). The upper bound in (31) is a consequence of the positivity of the free-energy. Finally, Eq. (32) follows from the fact that the thermal state $\rho_{B}$ is a minimum of the standard free energy $F_{T}(\rho)=$ $E(\rho)-T S(\rho)$, which implies $\Delta F_{T, B}=O\left(\delta \rho_{B}^{2}\right)$, and $\Delta E_{B}=T \Delta S_{B}+O\left(\delta \rho_{B}^{2}\right)$.

Now that heat has been unambiguously defined, we can pass to the definition of work (cf. [2]).

Definition 12 (Work). For an arbitrary entropy preserving transformation involving a system $A$ and its environment $B, \rho_{A B} \rightarrow \rho_{A B}^{\prime}$, with fixed non-interacting Hamiltonians $H_{A}$ and $H_{B}$, the worked performed on the system $A$ is defined as

$$
\Delta W_{A}:=W-\Delta F_{B},
$$

where $W=\Delta E_{A}+\Delta E_{B}$ is the work cost of implementing the global transformation and $\Delta F_{B}=$ $F\left(\rho_{B}^{\prime}\right)-F\left(\rho_{B}\right)$ is the change in free energy of the bath.

Equipped with the notions of heat and work, the first law takes the form of a mathematical identity.
Lemma 13 (First law). For an arbitrary entropy preserving transformation involving a system $A$ and its environment $B, \rho_{A B} \rightarrow \rho_{A B}^{\prime}$, with fixed non-interacting Hamiltonians $H_{A}$ and $H_{B}$, the change in energy for $A$ is distributed as

$$
\Delta E_{A}=\Delta W_{A}-\Delta Q .
$$

Proof. The proof follows from the definitions of work and heat.

The interpretation of this identity is that under a given process, $-\Delta W_{A}$ is the amount of energy that can be transferred to and stored in a battery. While the heat $\Delta Q_{A}$ is the change in energy due to flow of information from/into the system.

\section{Second law}

The second law of thermodynamics is formulated in many different forms: as an upper bound on the extracted work, the impossibility of converting heat into work completely, etc. In this section we show how all these formulations are a consequence of the principle of information conservation. Note that the formulation of the second law following from global entropy conservation is not new (e.g. in [39]), but it was thought to require a bath with well-defined temperature. What is new in the present approach is that we do not need even a bath, let alone that it is initially in a thermal state with pre-defined temperature.

One of the most important questions in thermodynamics, both historically and conceptually, is to which extent the energy in a heat bath can be converted into work. The second law puts a limit on the amount of work extracted. Over the years, research has led to various formulations of second law in standard thermodynamics, like the Clausius statement, the Kelvin-Planck statement and the Carnot statement, to mention a few.

Similar questions can be posed in the framework considered here, in terms of bound energy. In the following, we present analogous forms of second laws that consider these questions qualitatively as well as quantitatively.

\subsection{Work extraction}

One of the major concerns in thermodynamics is the conversion of any form of energy into work, which can be used for any application with certainty. 
Lemma 14 (Second law: work extraction). For an arbitrary composite system $\rho$, the extractable work by any entropy preserving process $\rho \rightarrow \rho^{\prime}$, $W=E(\rho)-E\left(\rho^{\prime}\right)$ is upper-bounded by the free energy

$$
W \leq F(\rho),
$$

which is saturated with equality if and only if $\rho^{\prime}=$ $\gamma(\rho)$.

If the system initially is in the particular state $\rho=\rho_{A} \otimes \gamma_{B}\left(T_{B}\right)$ with $\gamma_{B}\left(T_{B}\right)$ being thermal at temperature $T_{B}$, then

$$
W \leq F_{T_{B}}\left(\rho_{A}\right)-F_{T_{B}}\left(\gamma_{A}\left(T_{B}\right)\right)
$$

where $F_{T}(\cdot)$ is the standard out-of-equilibrium free energy, and the inequality is saturated in the limit of an infinitely large bath (infinite heat capacity).

Proof. By using $E\left(\rho^{\prime}\right) \geq B\left(\rho^{\prime}\right)$, one gets

$$
W=E(\rho)-E\left(\rho^{\prime}\right) \leq E(\rho)-B\left(\rho^{\prime}\right)=F(\rho),
$$

where note that $B\left(\rho^{\prime}\right)=B(\rho)$.

When the initial state has the composite structure $\rho=\rho_{A} \otimes \gamma_{B}\left(T_{B}\right)$, we get

$$
\begin{aligned}
W & \leq F\left(\rho_{A} \otimes \gamma_{B}\left(T_{B}\right)\right) \\
& \leq F_{T}\left(\rho_{A} \otimes \gamma_{B}\left(T_{B}\right)\right)-F_{T}\left(\gamma_{A}\left(T_{B}\right) \otimes \gamma_{B}\left(T_{B}\right)\right) \\
& =F_{T}\left(\rho_{A}\right)-F_{T}\left(\gamma_{A}\left(T_{B}\right)\right),
\end{aligned}
$$

we have used Lemma 5.

Finally, in the limit of an infinitely large bath, the intrinsic temperature $T_{A B}$ tends to the bath temperature $T_{B}$ and $\Delta Q=T_{B} \Delta S_{B}$, which saturates the bound in Eq. (38).

To see that the intrinsic temperature $T_{A B}$ tends to $T_{B}$ in the limit of large baths, let us increase the bath size by adding several copies of it. The entropy change of such bath reads

$$
\begin{aligned}
\Delta S_{B} & =S\left(\gamma_{B}^{\otimes n}\left(T_{A B}\right)\right)-S\left(\gamma_{B}^{\otimes n}\left(T_{B}\right)\right) \\
& =n\left(S\left(\gamma_{B}\left(T_{A B}\right)\right)-S\left(\gamma_{B}\left(T_{B}\right)\right)\right) .
\end{aligned}
$$

By considering the bound $\Delta S_{B}=-\Delta S_{A} \leq|A|$, we get

$$
S\left(\gamma_{B}\left(T_{A B}\right)\right)-S\left(\gamma_{B}\left(T_{B}\right)\right) \leq \frac{|A|}{n},
$$

which, together with the continuity of $S(\gamma(T))$, proves $\lim _{n \rightarrow \infty} T_{A B}=T_{B}$.

\subsection{Clausius statement}

The second law of thermodynamics not only dictates the direction of state transformations, but also establishes a fundamental bound on the amount of work that can be extracted by them. Here we first concentrate on the bounds on extractable work and introduce analogous versions of the second law in our setup.

Lemma 15 (Second law: Clausius statement). Any iso-entropic process involving two bodies $A$ and $B$ in an arbitrary state, with intrinsic temperatures $T_{A}$ and $T_{B}$, respectively satisfies the following inequality:

$\left(T_{B}-T_{A}\right) \Delta S_{A} \geqslant \Delta F_{A}+\Delta F_{B}+T_{B} \Delta I(A: B)-W$,

where $\Delta F_{A / B}$ is the change in the free energy of the body $A / B, \Delta I(A: B)$ is the change of mutual information, and $W=\Delta E_{A}+\Delta E_{B}$ is the amount of external work performed on the total system.

In the absence of initial correlations between the two bodies $A$ and $B$, the states being initially thermal, and no external work performed, this implies

$$
\left(T_{B}-T_{A}\right) \Delta S_{A} \geqslant 0,
$$

meaning that no iso-entropic equilibration process is possible whose sole result is the transfer of heat from a cooler to a hotter body.

Proof. The definition of free and bound energy implies that

$$
W=\Delta F_{A}+\Delta F_{B}+\Delta Q_{A}+\Delta Q_{B},
$$

where have used the definition of heat as the change of bound energy of the environment. From Eq. (31), one gets,

$$
\Delta Q_{A}+\Delta Q_{B} \geqslant T_{B} \Delta S_{B}+T_{A} \Delta S_{A},
$$

where $T_{A / B}$ is the initial intrinsic temperature of the body $A / B$. Due to the conservation of the total entropy, the change in mutual information can be written as $\Delta I(A: B)=\Delta S_{A}+\Delta S_{B}$. Putting everything together and noting that $\operatorname{sign}(\Delta B)=$ $\operatorname{sign}(\Delta S)$ completes the proof.

The terms on the right hand side of Eq. (41) show the three reasons for which the standard Clausius statement can be violated. Either because of the process not being spontaneous (external work is performed, $W>0$ ), or due to initial states having free 
energy which is consumed, or the presence of correlations [19].

An alternative formulation of the Clausius statement, for initial and final equilibrium states, is considered in Appendix A.

\subsection{Kelvin-Planck statement}

While the Clausius statement tells us that heat cannot flow spontaneously from a hotter to a colder body, the Kelvin-Plank formulation of second law states that, when heat going from a hotter to a colder body, it cannot be completely transformed into work.

Lemma 16 (Second law: Kelvin-Planck statement). Any iso-entropic process involving two bodies $A$ and $B$ in an arbitrary state satisfies the following energy balance

$$
\Delta Q_{B}+\Delta Q_{A}=-\left(\Delta F_{A}+\Delta F_{B}\right)+W,
$$

where $\Delta F_{A / B}$ is the change in the free energy of the body $A / B, \Delta Q_{A / B}$ the heat dissipated by the body $A / B$, and $W=\Delta E_{A}+\Delta E_{B}$ is the amount of external work performed on the total system.

In the case of the reduced states being thermal, and for a work extracting process $W<0$, the above equality becomes

$$
\Delta Q_{B}+\Delta Q_{A} \leqslant W<0 .
$$

Finally, in the absence of initial correlations, Eq. (46) implies that no iso-entropic equilibration process is possible whose sole result is the absorption of bound energy (heat) from an equilibrium state and its complete conversion into work.

Proof. Eq. (45) is a consequence of the energy balance (43). Eq. (46) follows from (45) by considering reduced states that are initially thermal and thereby $\Delta F_{A / B} \geqslant 0$. To prove the final statement is sufficient to notice that entropy preserving processes on initially uncorrelated systems fulfill $\Delta S_{A}+\Delta S_{B} \geqslant 0$, which together with (46) implies that $\operatorname{sign}\left(\Delta Q_{A}\right)=-\operatorname{sign}\left(\Delta Q_{B}\right)$.

An alternative formulation of Kelvin-Planck statement, for initial and final equilibrium states, is considered in Appendix B.

\subsection{Carnot statement}

A traditional heat engine extracts work from a situation in which two thermal baths $A$ and $B$ have different temperatures $T_{A}$ and $T_{B}$. The work extraction is usually implemented in practice by means of a working body $S$ which cyclically interacts with $A$ and $B$. Here we are not concerned with how precisely the working medium interacts with the baths $A$ and $B$, but just assume that at the end of every cycle the working body is left in its initial state and uncorrelated with the two bath. In other words, the working body only has the role to move energy from one bath to the other. From this perspective, the heat engine can be analyzed by studying the changes of the environments $A$ and $B$. In contrast to the standard situation, here we will not assume that the baths are infinitely large, but that they have a similar size as the system, and so the loss or gain of energy changes their (intrinsic) temperature.

Without loss of generality we assume $T_{A}<T_{B}$ and $\rho_{A B}=\gamma_{A}\left(T_{A}\right) \otimes \gamma_{B}\left(T_{B}\right)$. After operating the machine for one or several complete cycles, the baths experience a change $\rho_{A B} \rightarrow \rho_{A B}^{\prime}$.

We define the efficiency of work extraction in a heat engine as the fraction of energy that is taken from the hot bath which is transformed into work:

$$
\eta:=\frac{W}{-\Delta E_{B}},
$$

where $-\Delta E_{B}=E_{B}-E_{B}^{\prime}>0$ is the energy taken from the hot bath, and $W$ is the work extracted. In the following, we upper-bound the efficiency of any heat engine.

Lemma 17 (Second law: Carnot statement). For an engine working with two initially uncorrelated environments, $\rho_{A B}=\gamma_{A}\left(T_{A}\right) \otimes \gamma_{B}\left(T_{B}\right)$, each in a local equilibrium state with intrinsic temperatures $T_{B}>T_{A}$, the efficiency of work extraction is bounded by

$$
\eta \leqslant 1-\frac{\Delta B_{A}}{-\Delta B_{B}},
$$

where $\Delta B_{A}$ and $\Delta B_{B}$ are the changes in bound energies of the systems $A$ and $B$, respectively.

In the limit of large baths and under global entropy preserving operations, the Carnot efficiency is recovered,

$$
\eta \leqslant 1-\frac{T_{A}}{T_{B}} .
$$

Proof. The maximum extractable work due to the transformation $\rho_{A B} \rightarrow \rho_{A B}^{\prime}$ is given by

$$
W=F\left(\rho_{A B}\right)-F\left(\rho_{A B}^{\prime}\right)=\left(-\Delta E_{B}\right)-\Delta E_{A}>0 .
$$

The efficiency then reads

$$
\eta=1-\frac{\Delta E_{A}}{-\Delta E_{B}} .
$$


The condition of $A$ being initially at equilibrium implies that $\Delta F_{A} \geqslant 0$, from which it follows that $\Delta E_{A}>\Delta B_{A}$, and analogously for $B$. Thus,

$$
\eta \leqslant 1-\frac{\Delta B_{A}}{-\Delta B_{B}} .
$$

In the limit of large baths, in which $\Delta B_{A} \ll B_{A}$, the change in bound energy becomes $\Delta B_{A}=$ $T_{A} \Delta S_{A}$. Hence,

$$
\eta \leqslant 1-\frac{T_{A} \Delta S_{A}}{-T_{B} \Delta S_{B}}
$$

If the process is globally entropy preserving, i.e. $S_{A B}^{\prime}=S_{A}+S_{B}$, then $\Delta S_{A}+\Delta S_{B} \geqslant 0$, or alternatively $\Delta S_{A} \geqslant-\Delta S_{B}$, which together with (53) implies (49).

\section{Third law?}

The third law of thermodynamics establishes the impossibility of attaining the absolute zero temperature. According to Nernst, "it is impossible to reduce the entropy of a system to its absolute-zero value in a finite number of operations". The third law of thermodynamics has been very recently proven in a resource theoretic setting [50]. The unattainability of absolute zero entropy is a consequence of the unitary character of the transformations considered. For instance, consider the state transformation

$$
\rho_{S} \otimes \rho_{B} \longrightarrow|0\rangle\langle 0| \otimes \rho_{B}^{\prime},
$$

where $\rho_{B}$ is the thermal state of the bath $B$, which models the cooling down (erasure) of the system $S$, initially in a state $\rho_{S}$, which we assume to have $\operatorname{rank}\left(\rho_{S}\right)>1$. Here the dimension of the Hilbert space of the bath, $d_{B}$, is considered to be arbitrarily large but finite. As $\rho_{B}$ is a full-rank state, the left hand side and the right hand side of Eq. (54) have different ranks, and they cannot be transformed via a unitary transformation. Thus, irrespective of work supply, one cannot attain the absolute zero entropy state.

The zero entropy state can only be achieved for infinitely large baths and a sufficient work supply. Assuming a locality structure for the bath's Hamiltonian, this would take an infinitely long time. However, in the finite dimensional case, a quantitative bound on the achievable temperature given a finite amount of resources (e. g. work, time) is derived in [50].
Note that our set of entropy preserving operations is more powerful than unitaries. In particular, transformation (54) is always possible as long as the entropy of the system can be allocated in the bath, i.e.

$$
S\left(\rho_{S}\right) \leqslant \log d_{B}-S\left(\rho_{B}\right) .
$$

The required work to do so will be $W=F(|0\rangle\langle 0| \otimes$ $\left.\rho_{B}^{\prime}\right)-F\left(\rho_{S} \otimes \rho_{B}\right)$. As it has been discussed in Sec. 2, entropy preserving operations can be implemented as global unitaries acting on asymptotically many copies. Thus, the attainability of the absolute zero entropy state by means of entropy preserving operations is in agreement with the cases of infinitely large baths and unitary operations [50].

In conclusion, the third law of thermodynamics is a consequence of the microscopy reversibility (unitarity) of the transformation and is not respected by entropy preserving operations.

\section{A temperature-independent re- source theory of thermodynamics}

In this section we connect the framework developed above with the recently developed "standard" approach to thermodynamics as a resource theory. The main ingredients of a resource theory are the state space, which is usually compatible with a composition operation - for instance, quantum states together with the tensor product-, and a set of of allowed operations, which should facilitate state transformations that are in general irreversible. In a first attempt to have a resource theory of thermodynamics from our framework, it would seem that we are bound to transformations within an equivalence class, that is, between states on the same Hilbert space and with equal entropies, which is of course a very poor perspective. To compare and explore the interconvertibility of states with different entropies, it is necessary to extend the set of operations, and in particular to add a composition rule. In order to be able to connect states with different entropies and/or number of particles it is natural to consider different number of copies, and ask about rates of transformations.

Let us restrict first to the case in which the set of operations is entropy preserving regardless of the energy. We ask what is the rate of transforming $\rho$ into $\sigma$ and consider first the case that $S(\rho) \leq S(\sigma)$. Then, for large enough $n$ and $m$ we have

$$
S\left(\rho^{\otimes n}\right) \sim S\left(\sigma^{\otimes m}\right) .
$$


With such a trick of considering a different number of copies, we have brought $\rho$ and $\sigma$, which had different entropies, into the same manifold of equal entropy. There is a subtlety here which is that $\rho^{\otimes n}$ and $\sigma^{\otimes m}$ live in general on different Hilbert spaces. This can be easily circumvented by adding some product state, i.e.

$$
\rho^{\otimes n} \longrightarrow \sigma^{\otimes m} \otimes|0\rangle\left\langle\left. 0\right|^{\otimes(n-m)},\right.
$$

where $\rho^{\otimes n}$ and $\sigma^{\otimes m} \otimes|0\rangle\left\langle\left. 0\right|^{\otimes(n-m)}\right.$ now live on the same Hilbert space. Eq. (57) can also be understood as a process of information compression, in which the information in $n$ copies of $\rho$ is compressed to $m<n$ copies of $\sigma$, and $n-m$ systems have been erased. Thus, in the case of having only an entropy preserving constraint, the transformation rate can be determined from $(56)$

$$
r:=\lim _{n \rightarrow \infty} \frac{m}{n}=\frac{S(\rho)}{S(\sigma)} .
$$

In thermodynamics, however, energy must also be taken into account. When one does that, it could well be that the process (57) is not energetically allowed, since it might happen that $E\left(\rho^{\otimes n}\right) \neq E\left(\sigma^{\otimes m}\right)$. In such a case, we would need more copies of $\rho$, in order for the transformation to be energetically favorable. This would force us to add an entropic ancilla, since otherwise, the initial and final state of such a process would not have the same entropy.

Let us thus consider a transformation

$$
\rho^{\otimes n} \longrightarrow \sigma^{\otimes m} \otimes \phi^{\otimes(n-m)},
$$

where the number of copies $n$ and $m$ have to fulfil the energy and entropy conservation constraints

$$
\begin{aligned}
E\left(\rho^{\otimes n}\right) & \sim E\left(\sigma^{\otimes m} \otimes \phi^{\otimes(n-m)}\right), \\
S\left(\rho^{\otimes n}\right) & \sim S\left(\sigma^{\otimes m} \otimes \phi^{\otimes(n-m)}\right) .
\end{aligned}
$$

The above conditions can be easily written as a geometric equation of the points $x_{\psi}=(E(\psi), S(\psi))$ with $\psi \in\{\rho, \sigma, \phi\}$ in the energy-entropy diagram:

$$
x_{\rho}=r x_{\sigma}+(1-r) x_{\phi},
$$

where $r:=m / n$ is the conversion rate, and we have only used the extensivity of both entropy and energy in the number of copies, e.g. $E\left(\rho^{\otimes n}\right)=n E(\rho)$.

Eq. (61) implies that the three points $x_{\rho, \sigma, \phi}$ need to be aligned. In addition, the fact that $0 \leq r \leq$ 1 implies that $x_{\rho}$ lies in the segment connecting $x_{\sigma}$ and $x_{\phi}$ (see Fig. 6). The conversion rate $r$ has then a geometric interpretation. It is the relative Euclidean

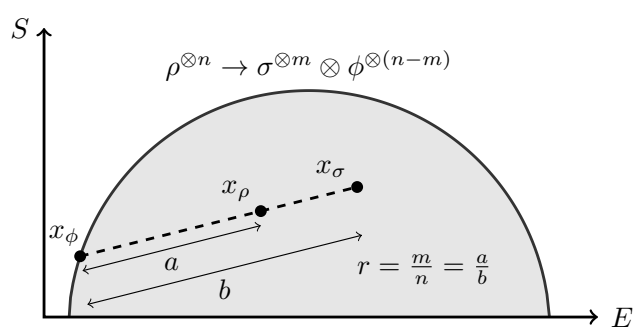

Figure 6: Representation in the energy-entropy diagram of how the energy and entropy conservation constraints in the transformation $\rho^{\otimes n} \rightarrow \sigma^{\otimes m} \otimes \phi^{\otimes n-m}$ imply $x_{\rho}$, $x_{\sigma}$ and $x_{\phi}$ to be aligned and $x_{\rho}$ to lie in between $x_{\sigma}$ and $x_{\phi}$.

distance between $x_{\phi}$ and $x_{\rho}$ over the total distance $\left\|x_{\sigma}-x_{\phi}\right\|$, or in other words, it is the fraction of the path that one has run when going from $x_{\phi}$ to $x_{\sigma}$ and reaches $x_{\rho}$ (see Fig. 6).

In order for the conversion rate $r$ from $\rho$ and $\sigma$ to be maximum, the state $\phi$ needs to lie in the boundary of the energy-entropy diagram, that is, it needs to be either a thermal or a pure state. This allows us to quantitatively determine the conversion rate $r$ by first finding the temperature of the thermal state $\phi$ which satisfies

$$
\frac{S(\sigma)-S(\phi)}{S(\sigma)-S(\rho)}=\frac{E(\sigma)-E(\phi)}{E(\sigma)-E(\rho)},
$$

and then

$$
r=\frac{S(\rho)-S(\phi)}{S(\sigma)-S(\phi)}
$$

See the geometrical interpretation in Fig. 6.

Note how Eq. (63) becomes (58) in the case of $\phi$ being pure. Let us also mention that, although at the first sight the inter-convertibility rate (63) looks different from the one obtained in [43], it can be proven that they are the same. Eq. (63) is more compact than the one given there, and its derivation much less technical.

\section{Thermodynamics with multiple conserved quantities}

The formalism for thermodynamics developed above can easily be extended to situations with multiple conserved quantities besides the energy, which we call "charges". These could be particle numbers of certain chemical species, or physically conserved quantities such as angular momentum. Such extensions to quantum thermodynamics, especially in the resource setting, have been considered previously by several authors [51-55]. 


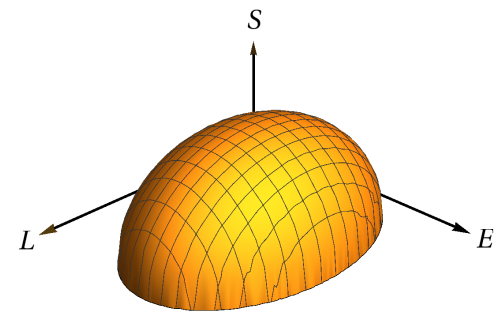

Figure 7: Schematic of the charges-entropy diagram of a system with two conserved quantities $E$ and $L$.

The setting is that each system comes with $q$ conserved quantities $\hat{L}_{k}$ for $k=0, \ldots, q-1$, with $\hat{Q}_{0}=$ $H$ the Hamiltonian. Here we will assume that these quantities are all pairwise commuting. As before, when we compose systems, the quantities are simply added: for another Hilbert space $\mathcal{H}^{\prime}$ with charges $\hat{L}_{k}^{\prime}$ $(k=0, \ldots, q-1)$, the joint Hilbert space $\mathcal{H} \otimes \mathcal{H}^{\prime}$ carries the global (or total) charges $\hat{L}_{k} \otimes \mathbb{1}+\mathbb{1} \otimes \hat{L}_{k}^{\prime}$.

\subsection{The charges-entropy diagram}

The energy-entropy diagram from the previous discussion is extended to include the additional conserved quantities. Let us introduce the chargesentropy diagram as:

Definition 18 (Charges-entropy diagram). The charges-entropy diagram is the subset of points in $\mathbb{R}^{q+1}$ produced by all the states $\rho \in \mathcal{B}(\mathcal{H})$ with coordinates

$$
x_{\rho}=\left(L_{0}(\rho), L_{1}(\rho), \ldots, L_{q-1}, S(\rho)\right),
$$

where $L_{k}(\rho):=\operatorname{Tr} \hat{L}_{k} \rho$ for $k=0, \ldots, q-1$. Note that in this section, to avoid confusion between the observable (the operator $\hat{L}_{k}$ ) and the coordinate in the charges-entropy diagram (the number $L_{k}$ ), we mark the former always by a hat.

Using the mutual commutation of the conserved quantities, we can easily show that the chargesentropy diagram forms a convex set in $\mathbb{R}^{q+1}$. To do so, let us first prove convexity for the zero entropy hyperplane, which corresponds to the base of the diagram. The zero entropy hypersurface of the chargesentropy diagram is the set of points in $\mathbb{R}^{q}$ with coordinates $x_{\psi}=\left(L_{0}(\psi), L_{1}(\psi), \ldots, L_{q-1}(\psi)\right)$ for all normalized pure states $\psi=|\psi\rangle\langle\psi|$. Let us consider in $\mathbb{R}^{q}$ the family of hyperplanes perpendicular to the unit vector $\vec{\mu}=\left(\mu_{0}, \ldots, \mu_{q-1}\right)$

$$
\vec{\mu} \cdot \vec{L}=\sum_{k=0}^{q-1} \mu_{k} L_{k}=C,
$$

where $\vec{L}=\left(L_{0}, \ldots, L_{q-1}\right)$ is a point in $\mathbb{R}^{q}$ and $C$ determines the hyperplane. All points with coordinates $\vec{L}$ that fulfil Eq. (65) belong to the hyperplane defined by $\vec{\mu}$ and $C$. Given a direction $\vec{\mu}$, there is an hyperplane that is particularly relevant, since it corresponds to the minimum possible value of $C$

$$
C_{\min }(\vec{\mu})=\min _{\psi \in \mathcal{H}} \frac{\langle\psi|\vec{\mu} \cdot \overrightarrow{\hat{L}}| \psi\rangle}{\langle\psi \mid \psi\rangle}=\min _{\psi \in \mathcal{H}} \frac{\vec{\mu} \cdot \vec{L}(\psi)}{\langle\psi \mid \psi\rangle} .
$$

The minimum is reached by the eigenstate with smallest eigenvalue of the Hermitian operator $\vec{\mu} \cdot \hat{L}$ that we denote by $|\psi(\vec{\mu})\rangle$,

$$
\left(\sum_{k} \mu_{k} \hat{L}_{k}\right)|\psi(\vec{\mu})\rangle=C_{\min }(\vec{\mu})|\psi(\vec{\mu})\rangle .
$$

Assume for the moment that there is a unique ground state of $\vec{\mu} \cdot \overrightarrow{\hat{L}}$. This means that the hyperplane defined by $C_{\min }(\vec{\mu})$ is tangent to the charges-entropy diagram since it passes through one point of the diagram (corresponding to the single ground state $\mid \psi(\vec{\mu}\rangle)$ ) leaving the rest of the diagram on the same side, i.e. $\vec{\mu} \cdot \vec{L}(\psi) \geqslant C_{\min }(\vec{\mu})$ for all $|\psi\rangle \in \mathcal{H}$.

In case that there is a degenerate ground space described by a basis whose elements have different coordinates in the space of charges, the contact between the hyperplane $C=C_{\min }$ and the zero entropy hypersurface is not a point but a simplex of some higher dimension, up to the dimension of the ground space (i.e. a segment in dimension 2 , triangle in dimension 3 , etc). As the conserved quantities are mutually commuting, there is a common eigenbasis for all the $q$ charges. Thus, there exists a basis of the ground space of $\vec{\mu} \cdot \vec{L}$ which is also eigenbasis of all the charges $L_{k}$ individually. If $|\psi\rangle$ and $\left|\psi^{\prime}\right\rangle$ are two elements of that basis with different coordinates $\vec{L}$ and $\vec{L}^{\prime}$, the state $|\psi(\theta)\rangle=\cos \theta|\psi\rangle+\sin \theta\left|\psi^{\prime}\right\rangle$ with $\theta \in[0, \pi / 2]$ has coordinates

$$
\vec{L}(\psi(\theta))=\left(\cos ^{2} \theta\right) \vec{L}+\left(\sin ^{2} \theta\right) \vec{L}^{\prime},
$$

that is, the coordinates of $\psi(\theta)$ in the diagram are simply the corresponding convex combinations of the coordinates of $\vec{L}$ and $\vec{L}^{\prime}$. 
Property (68) holds for any two eigenstates of $\overrightarrow{\hat{L}}$, in particular, for any two states $|\psi\rangle$ and $\left|\psi^{\prime}\right\rangle$ that lie in the boundary of the zero-entropy hypersurface. Hence, as any point in the bulk of the zero-entropy hypersurface can be obtained as a convex combination of two points of the boundary, all the points in the bulk belong to the zero entropy surface. Altogether, this proves that the zero-entropy surface is a convex set.

Once we understand the zero entropy hypersurface of the charges-entropy diagram, let us study its upper boundary. The upper boundary of the charges-entropy diagram is described by the Generalized Gibbs Ensemble (GGE) instead of the canonical ensemble, i.e.

$$
\gamma(\vec{\beta}):=\frac{1}{Z_{\vec{\beta}}} \mathrm{e}^{-\sum_{k} \beta_{k} \hat{L}_{k}},
$$

where $Z_{\vec{\beta}}:=\operatorname{Tr}\left(\mathrm{e}^{-\sum_{k} \beta_{k} \hat{L}_{k}}\right)$ is the generalized partition function. These states are precisely the states of maximum entropy among all those with prescribed expectation values $\left\langle\hat{L}_{k}\right\rangle, k=0, \ldots, q-1$. We call this boundary the equilibrium boundary.

The equilibrium boundary is mathematically described by all the points $\vec{L}(\rho)$ for which there exists a $\vec{\beta} \in \mathbb{R}^{q}$ such that

$$
\sum_{k=0}^{q-1} \beta_{k} L_{k}(\rho)-S(\rho)=-\log Z_{\beta} .
$$

Note that, given some $\vec{\beta}$, the points $\vec{L}(\rho)$ that fulfil

$$
\sum_{k=0}^{q-1} \beta_{k} L_{k}(\rho)-S(\rho)=C,
$$

belong to a hyperplane of dimension $q$. The hyperplane with $C=-\log Z_{\beta}$ is the one tangent to the equilibrium boundary. The normal vector to this family of hyperplanes $(71)$ is precisely $(\vec{\beta},-1)$. This agrees with the fact that the tangent plane has a slope $\beta_{k}$ in the $k$-th direction, i. e.

$$
\frac{\partial S}{\partial L_{k}}=\beta_{k} \quad \forall k=0, \ldots, q-1 .
$$

Eq. (72) can also be written in a vectorial form as

$$
\vec{\beta}=\vec{\nabla} S,
$$

and $\vec{\beta}$ corresponds to the direction of maximal variation of the entropy. This is shown in Fig. 8.

Note that in order to microscopically justify the charges-entropy diagram, it must be shown that states

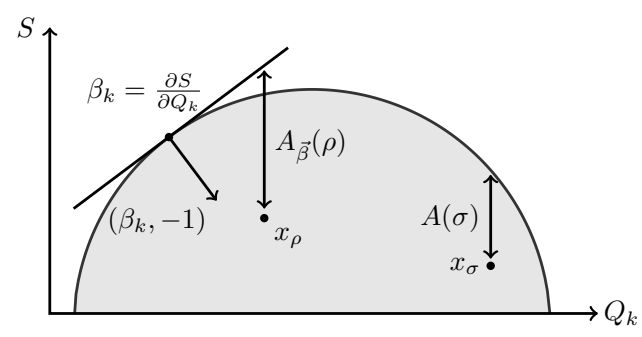

Figure 8: Transverse section of the charges-entropy diagram in the $L_{k}-S$ plane. The normal vector to the tangent plane has coordinates $(\vec{\beta},-1)$, which in the section appears as $\left(\beta_{k},-1\right)$. The $\vec{\beta}$-athermality, $A_{\vec{\beta}}(\rho)$, and the absolute athermality, $A(\sigma)$, are respectively represented for two states $\rho$ and $\sigma$.

with the same expectation values for the conserved quantities can be connected to each other using unitaries that commute with all charges, and using sublinear ancillas, in the limit of many copies. To state the theorem, we fix some notation. Consider a quantum system with mutually commuting charges $\hat{L}_{0}=H, \hat{L}_{1}, \ldots, \hat{L}_{q-1}$ on a finite dimensional Hilbert space $\mathcal{H}$. The corresponding description of $n$ copies of this system has Hilbert space $\mathcal{H}^{\otimes n}$ and charges

$$
\hat{L}_{k}^{(n)}=\sum_{i=1}^{n} \mathbb{1}_{\mathcal{H}}^{\otimes i-1} \otimes\left(\hat{L}_{k}\right)_{i} \otimes \mathbb{1}_{\mathcal{H}}^{\otimes n-i} .
$$

Theorem 19. Two states $\rho$ and $\sigma$ of the above quantum system have the same entropy $S(\rho)=$ $S(\sigma)$ and the average charge values $\operatorname{Tr}\left(\hat{Q}_{k} \rho\right)=$ $\operatorname{Tr}\left(\hat{Q}_{k} \sigma\right)$ for all $k=0, \ldots, q-1$ if and only if for all $n$ there exists an ancillary quantum system with the Hilbert space $\mathcal{K}$ of dimension $2^{o(n)}$, with charges $\hat{L}_{0}^{\prime}=H^{\prime}, \hat{L}_{1}^{\prime}, \ldots, \hat{L}_{q-1}^{\prime}$, and a charge conserving unitary $U$ acting on $\mathcal{H}^{\otimes n} \otimes \mathcal{K}$, such that

$$
\begin{aligned}
& \lim _{n \rightarrow \infty}\left\|U\left(\rho^{\otimes n} \otimes \omega^{\prime}\right) U^{\dagger}-\sigma^{\otimes n} \otimes \omega\right\|_{1}=0, \quad \\
& \left\|\hat{L}_{k}^{\prime}\right\|_{\infty} \leq o(n) \text { and }\left[U, \hat{L}_{k}^{(n)}+\hat{L}_{k}^{\prime}\right]=0 \quad \forall k,
\end{aligned}
$$

where $\omega$ and $\omega^{\prime}$ are suitable states of the ancillary system.

The proof of this theorem (which generalises the asymptotic equivalence theorem of [43]) is presented in our forthcoming work [56]. There, we extensively study this issue, and show how to generalize it 
even further from commuting to non-commuting conserved charges. In the following, we limit ourselves to the main points.

\subsection{Athermality and free entropy}

Proceeding as before, let us introduce some quantities that will be relevant in the following.

Definition 20 ( $\vec{\beta}$-athermality). The $\vec{\beta}$ athermality of a state $\rho$ is defined as

$$
A_{\vec{\beta}}(\rho):=\sum_{k=0}^{q-1} \beta_{k} L_{k}(\rho)-S(\rho)+\log Z_{\vec{\beta}} .
$$

The $\vec{\beta}$-athermality of a point with coordinates $(\vec{L}(\rho), S(\rho))$ can be interpreted geometrically in the charges-entropy diagram as the vertical distance from the hyperplane tangent to the equilibrium boundary with normal vector $(\vec{\beta},-1)$. This is represented in Fig. 8 . Note that the $\vec{\beta}$-athermality is also introduced in [54] under the name free entropy.

The $\vec{\beta}$-athermality can also be written as

$$
A_{\vec{\beta}}(\rho)=D(\rho \| \gamma(\vec{\beta}))
$$

with $D(\rho \| \sigma):=\operatorname{Tr}(\rho \log \rho-\rho \log \sigma)$ being the relative entropy. Hence, because of the non-negativity of the relative entropy $D(\rho \| \sigma) \geqslant 0$, we have that

$$
S(\rho) \leqslant \sum_{k=0}^{q-1} \beta_{k} L_{k}(\rho)+\log Z_{\vec{\beta}} .
$$

Note now that the right hand side corresponds to the entropy coordinate of the hyperplane with normal vector $\vec{\beta}$ tangent to the equilibrium boundary. Thus, any tangent hyperplane with normal vector $\vec{\beta}$ leaves all the points of the charges-entropy diagram below it. This is consistent with what we saw before, that the charges entropy diagram is a convex set.

In a similar way we define the absolute athermality or simply athermality in the following.

Definition 21 (Absolute athermality). The $a b$ solute athermality of a state $\rho$ is defined as

$$
A(\rho):=\min _{\vec{\beta} \in \mathbb{R}^{q}} A_{\vec{\beta}}(\rho) .
$$

The athermality of a state $\rho$ can be understood geometrically as the vertical distance from the thermal boundary (see Fig. 8).

\subsection{Charge extraction}

A first relevant scenario of thermodynamics with multiple conserved quantities is the extraction of a charge of the system while keeping the other charges constant. For this subset of operations, the system is restricted to move along a straight line in the direction of the extracted charge within the iso-entropic hyperplane (see Fig. 9).

The bound charge for such a scenario is given by

$$
B_{k}(\rho):=\min _{\substack{\sigma: S(\sigma)=S(\rho), L_{i}(\sigma)=L_{i}(\rho) \forall i \neq k}} L_{k}(\sigma),
$$

where $\gamma_{k}(\rho)$ is the GGE state that attains the minimum and corresponds to the point of the equilibrium boundary which is intersected by the straightline with direction $k$ which passes through $\rho$. As in the case of only energy, it is easy to see that $B_{k}(\rho)=L_{k}\left(\gamma_{k}(\rho)\right)$. This is represented in Fig. 9 (left). The direction $\vec{\beta}$ corresponding to $\gamma_{k}$ can be geometrically determined as the normal vector of the tangent plane to the equilibrium surface in that point.

The free charge $F_{k}(\rho)$ is now analogously defined as

$$
F_{k}(\rho)=L_{k}(\rho)-B_{k}(\rho),
$$

and corresponds to the maximum amount of charge that can be extracted given the restrictions of constant entropy and charges. This is represented in Fig. 9 (left) for a case of 2 conserved quantities.

A detailed and full rigorous analysis of the charge extraction by means of unitary conserving operations in the many copy limit (for both commuting and noncommuting charges) will be made in our forthcoming work [56].

\subsection{Extraction of a generalized potential}

An different, but also relevant scenario is when the set of operations allowed for the variation of the charges is unrestricted, and the aim is the extraction of a generalized potential; cf. [54, 55]

$$
V_{\vec{\mu}}(\rho):=\sum_{k=0}^{q-1} \mu_{k} L_{k}(\rho),
$$

where $\vec{\mu}:=\left(\mu_{0}, \ldots, \mu_{q-1}\right)$ specifies the weight $\mu_{k}$ of every charge $L_{k}$ in the linear combination $V_{\vec{\mu}}$. For convenience, $\vec{\mu}$ is assumed to be normalized $\|\vec{\mu}\|=1$ in the Euclidean norm and its components to be positive $\mu_{k} \geqslant 0$. (An alternative normalization could be to take the coefficient of the Hamiltonian $\mu_{0}=1$ as it is usually done in the grand canonical ensemble.) 

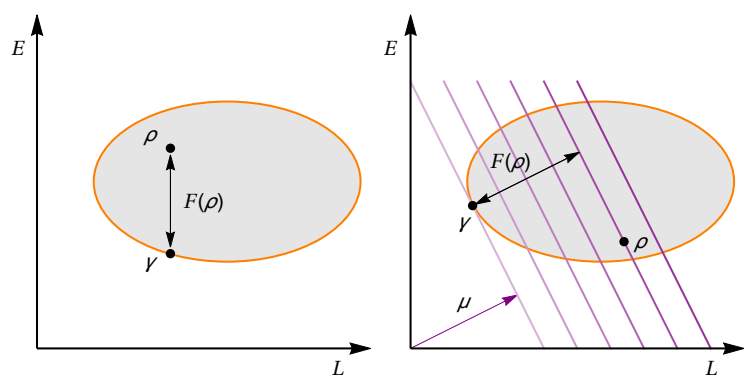

Figure 9: Constant entropy section of a charges-entropy diagram with 2 charges $E$ and $L$. (Left) Scenario of extraction of a single charge while keeping constant the other charges. The system is constrained to move in one dimensional line and free-charge $F(\rho)$ of a state $\rho$ is the distance from the boundary in such direction. (Right) Scenario in which the charges are allowed to change and the aim is the extraction of a potential $V_{\hat{\mu}}(\rho)=\sum_{k=0}^{q-1} \mu_{k} L_{k}(\rho)$. The parallel purple lines represent equipotential surfaces of $V_{\hat{\mu}}$. The GGE state $\gamma$ is the state which minimizes $V$ and belongs to the hyperplane which is tan tangent to the equilibrium boundary.

In this setting, the bound potential is defined as

$$
B_{\vec{\mu}}(\rho):=\min _{\sigma: S(\sigma)=S(\rho)} V_{\vec{\mu}}(\sigma),
$$

where $\gamma_{\vec{\mu}}(\rho)$ is the state that attains the minimum which is again a GGE. As before, $B_{\vec{\mu}}(\rho)=$ $V_{\vec{\mu}}\left(\gamma_{\vec{\mu}}(\rho)\right)$. Note that the $\vec{\beta}$ values of the state $\gamma_{\vec{\mu}}(\rho)$ need to be proportional to the unit vector $\vec{\mu}$

$$
\vec{\beta}=\beta \vec{\mu},
$$

with $\beta$ a scalar that is determined by the condition of equal entropies, $S(\rho)=S\left(\gamma_{\vec{\mu}}(\rho)\right)$. This can be seen geometrically in Fig. 9 (right) or analytically by making the simple observation that, regarding $\tilde{H}:=V_{\vec{\mu}}$ as a new Hamiltonian, the min-energy principle singles out $\mathrm{e}^{-\beta \tilde{H}}$ as the state that attains the minimum.

Analogously, the free potential is given by

$$
F_{\vec{\mu}}(\rho)=V_{\vec{\mu}}(\rho)-B_{\vec{\mu}}(\rho),
$$

and corresponds to the maximum amount of generalized potential $V_{\vec{\mu}}$ that can be extracted under entropy preserving operations. This situation has been diagrammatically represented in Fig. 9.

The scenario with a generalized potential $V_{\vec{\mu}}$ is analogue to the single charge situation. Both first and second laws can be stated as in Secs. 7 and 8, replacing the free energy $F(\rho)$ by $F_{\vec{\mu}}(\rho)$.

\subsection{Second law}

Any process (not necessarily entropy preserving) that brings an initial generalized Gibbs state $\gamma_{B}(\vec{\beta})$ out of equilibrium fulfils

$$
\sum_{k} \beta_{k} \Delta L_{k}^{B} \geqslant \Delta S_{B}
$$

where the inequality is only saturated in the limit of large baths (small variations of entropy) and when final state is also at equilibrium (GGE). Geometrically, Eq. (87) is a trivial consequence that the charges entropy diagram is upper bounded by any of its tangent planes.

In the particular case of an entropy preserving process on an initial bipartite state $\rho_{A} \otimes \gamma_{B}(\vec{\beta})$, the change in mutual information between the subsystems $A$ and $B$ is

$$
\Delta I=\Delta S_{A}+\Delta S_{B} \geqslant 0
$$

which together with Eq. (87) implies

$$
\sum_{k} \beta_{k} \Delta L_{k}^{B} \geqslant-\Delta S_{A}
$$

Note that the above equation (89) is equivalent to the second law formulated in [54]. We see here that such a law is also valid for baths of arbitrary small sizes. Eq. (89) is asymptotically saturated with equality in the limit of large system sizes and in the absence of correlations in the final state $\rho_{A B}^{\prime}=\rho_{A}^{\prime} \otimes \gamma_{B}^{\prime}$.

\subsection{Interconvertibility rates}

The rate of interconversion from a quantum state $\rho$ to a state $\sigma$, in the same sense as in Section 10, i.e.

$$
\rho^{\otimes n} \longrightarrow \sigma^{\otimes m} \otimes \phi^{\otimes(n-m)},
$$

is given by the conservation of the entropy and the charges $L_{k}$ :

$$
\begin{aligned}
L_{k}\left(\rho^{\otimes n}\right) & \sim L_{k}\left(\sigma^{\otimes m} \otimes \phi^{\otimes(n-m)}\right) \forall k=0, \ldots, q-1, \\
S\left(\rho^{\otimes n}\right) & \sim S\left(\sigma^{\otimes m} \otimes \phi^{\otimes(n-m)}\right) .
\end{aligned}
$$

The above conditions can again be written as a geometric equation for the points $x_{\xi}=$ $\left(L_{0}(\xi), \ldots, L_{q-1}(\xi), S(\xi)\right) \in \mathbb{R}^{q+1}$ in the energyentropy diagram, with $\xi \in\{\rho, \sigma, \phi\}$ :

$$
x_{\rho}=r x_{\sigma}+(1-r) x_{\phi},
$$


where we have merely used the extensivity of both entropy and the conserved quantities in the number of copies, e.g. $S\left(\rho^{\otimes n}\right)=n S(\rho)$.

Thus, by means of the same argument used in the previous section, the convertibility rate from $\rho$ to $\sigma$ reads

$$
r=\lim _{n \rightarrow \infty} \frac{m}{n}=\frac{S(\rho)-S(\phi)}{S(\sigma)-S(\phi)},
$$

where the state $\phi$ is determined from the following set of $q$ equations

$\frac{S(\sigma)-S(\phi)}{S(\sigma)-S(\rho)}=\frac{L_{k}(\sigma)-L_{k}(\phi)}{L_{k}(\sigma)-L_{k}(\rho)} \quad \forall k=0, \ldots, q-1$.

The state $\phi$ can be found geometrically in the charges-entropy diagram as the point in the boundary of the charges-entropy diagram that intersects the straight half-line that goes through $\rho$ from $\sigma$ (see Fig. 6 for the single charge example).

\section{Discussion}

In the recent years, thermodynamics, and in particular work extraction from non-equilibrium states, has been studied in the quantum domain, giving rise to radically new insights into quantum thermal processes. However, in the standard treatment of thermodynamics, be it classical or quantum, thermal baths are considered to be asymptotically large in size. That is why if a system is attached to a bath and allowed to exchange energy and entropy, the bath stays intact and its temperature remains unchanged. That is also why the equilibrated system finally acquires the same temperature as the bath. However, if one goes beyond this assumption and considers the bath to be a finite system, too, then traditional thermodynamics breaks down. This situation is very much relevant for thermodynamics in the quantum regime, where both system and bath may be small. The first problem that appears in such a situation is the notion of temperature itself, since the finite bath may go out of thermal equilibrium due to the exchange of energy with the system. Therefore, it is absolutely necessary to develop a temperature independent universal thermodynamics, in which the bath could be small or large, and would not get any special status.

In this work, we have formulated temperature independent thermodynamics as an exclusive consequence of information conservation. We have relied on the fact that, for a given amount of information, measured by the von Neumann entropy, any system can only be transformed to states with the same entropy. Given this constraint of information conservation, there is a distinguished state within the constant entropy manifold, which is the state that possesses minimal energy. This state is known as a completely passive state and acquires the BoltzmannGibbs canonical form with an intrinsic temperature. We call the energy of the completely passive state the bound energy, since no further energy can be extracted by means of entropy preserving operations. Thus, for a given state, the difference between its energy and bound energy corresponds to the maximum amount of energy that can be extracted in form of work and we call it free energy. In fact, in this framework, two states that have the same entropy and energy are thermodynamically equivalent [43]. The thermodynamic equivalence between equal entropy and energy states has allowed us to use of the energyentropy diagram to illustrate the notions of bound and free energies in a geometric way. We have introduced a new definition of heat for arbitrary systems in terms of the bound energy of the bath.

We have seen that the laws of thermodynamics are a consequence of the reversible dynamics of the underlying physical theory. In particular:

- Zeroth law emerges as the consequence of information conservation.

- First and second law emerge as the consequence of energy conservation, together with information conservation.

- Third law emerges as the consequence of finegrained information conservation (microscopic reversibility or unitarity). Therefore, there is no third law if one considers coarse-grained information conservation, as we did here.

We have demonstrated that the maximum efficiency of a quantum engine with a finite bath is in general lower than that of an ideal Carnot engine. We have introduced a resource theoretic framework for our intrinsic thermodynamics, within which we address the problem of work extraction and inter-state transformations. All these results have been illustrated by means of the energy-entropy diagram. Furthermore, we gave a geometric interpretation in the diagram of the relevant thermodynamic quantities, as well as the inter-convertibility rate between quantum states under entropy and energy preserving operations.

The information conservation based framework for thermodynamics, as well as the resource theory and 
the energy-entropy diagram, is also extended to multiple conserved quantities, as long as they commute with each other. In this case, the energy-entropy diagram becomes the charges-entropy diagram and allows us to understand thermodynamics in a geometrical way. In particular, we have studied the extraction of a single charge while keeping the other charges conserved as well as the extraction of a generalized potential. In the first scenario, we have seen that the maximum work extractable from any state by operations that asymptotically conserve the given charges is the difference between the free energy of the state and that of the iso-entropic generalized grand canonical Gibbs state. Concerning the extraction of a generalized potential (a linear combination of charges), we have shown that it is analogous to the work extraction (the single charge case). Finally, we have determined the interconvertibility rates between states with different entropy and charges. A deeper investigation of the setting with multiple charges, including the generalisation to non-commuting conserved quantities, is left to our forthcoming paper [56].

In general, thermodynamics can be studied in three different scenarios:

- One-shot or single-copy setting, where only one copy of the joint system-environment is available. In this case, even the notion of expectation value is not meaningful, as well as von Neumann entropy.

- Limit of many runs, where there are many copies, but operation are restricted to single copies of the system. In this scenario, the notions of expectation value and von Neumann entropy are well defined.

- Limit of many copies, where one has access to arbitrarily many copies of the system and an ancilla sub-linear in the number of copies that can globally be processed.

A first observation is that our formalism cannot be applied in the single-shot setting, since the notion of expectation value (say energy) cannot in general be used.

A relevant point to discuss is what happens when operations are not entropy preserving but unitaries. In the limit of many copies, state transformations under unitaries converge to entropy preserving operations and our formalism is recovered. The limit of many runs is a bit more subtle. On the one hand, all the thermodynamic inequalities of our formalism are respected, since unitaries form a subset of entropy preserving operations. On the other hand, these thermodynamic inequalities will not be in general saturated. For instance, our formalism states that the work that can be extracted per system in a state $\rho$ is upper bounded by its free energy $W \leqslant F(\rho)$. In the single-copy or many-run settings with fine-grained information conservation, the law will be respected, but there will not be in general a unitary for which $W=F(\rho)$.

A natural open question is to what extent our formalism can be extended from considering coarsegrained information conservation operations as the set of allowed operations to unitaries. In that case, the notion of bound energy would be different and many more equivalence classes of states would appear. Something similar already happens in the resource theory of thermodynamics, where instead of having a single monotone as an "if and only if" condition for state transformation, infinitely many are required [30]. It is far from clear whether under fine-grained information conservation restriction the energy-entropy diagrams (or a generalization of them) would still be useful.

Let us finally point out that, in the extension of our work to unitary operations in the settings of singleshot and many-runs, a consistent formulation of the zeroth law would not be possible. Recall that the zeroth law states that a collection of systems are in mutual thermal equilibrium if and only if their arbitrary combinations are also in equilibrium. It is well known that passive states that are not thermal do not remain passive when sufficiently many copies are considered. Hence, for establishing a consistent zeroth law, one has to consider operations beyond unitaries on a single copy.

\section{Acknowledgements}

We thank Ph. Faist, K. Gawędzki, R. B. T. Harvey, J. Kimble, S. Maniscalco, Ll. Masanes, J. Oppenheim, V. Pellegrini, M. Polini, C. Sparaciari, A. Vulpiani, N. Yunger Halpern and R. Zambrini for useful discussions and comments in both theoretical and experimental aspects of our work. We also thank the referees for constructive comments.

We acknowledge financial support from the European Commission (FETPRO QUIC H2020FETPROACT-2014 No. 641122), the European Research Council (AdG OSYRIS and AdG IRQUAT), the Spanish MINECO (grants no. FIS2008-01236, 
FISICATEAMO FIS2016-79508-P, FIS2013-40627P, FIS2016-86681-P, and Severo Ochoa Excellence Grant SEV-2015-0522) with the support of FEDER funds, the Generalitat de Catalunya (grants no. 2017 SGR 1341, and SGR 875 and 966), CERCA Program/Generalitat de Catalunya and Fundació Privada Cellex. AR thanks for support from the CELLEXICFO-MPQ fellowship.

\section{References}

[1] Jochen Gemmer, M. Michel, and Günther Mahler. Quantum Thermodynamics, volume 748 of Lecture Notes in Physics. Berlin, Heidelberg: Springer, 2009. ISBN 9783540705093. DOI: $10.1007 / 978-3-540-70510-9$. URL http://link. springer.com/book/10. 1007\%2F978-3-540-70510-9.

[2] Michael Flanders and Donald Swann. The First and Second Law of Thermodynamics. In At the Drop of Another Hat. Parlophone Ltd., 1964. URL https: //youtu. be/VnbiVw_1FNs.

[3] Juan M. R. Parrondo, Jordan M. Horowitz, and Takahiro Sagawa. Thermodynamics of information. Nature Physics, 11:131-139, 2015. DOI: doi:10.1038/nphys3230. URL http:// dx.doi.org/10.1038/nphys3230.

[4] James Clerk Maxwell. Theory of Heat. Longmans, Green, and Co.: London, New York, Bombay, 1908.

[5] Harvey S. Leff and Andrew F. Rex. Maxwell's Demon: Entropy, Information, Computing. Princeton University Press, 1990. ISBN 9780691605463. URL http://press. princeton.edu/titles/4731.html.

[6] Harvey S. Leff and Andrew F. Rex. Maxwell's Demon 2: Entropy, Classical and Quantum Information, Computing. Taylor and Francis, London, 2002. ISBN 9780750307598. URL https://www.taylorfrancis.com/books/ 9780750307598.

[7] Koji Maruyama, Franco Nori, and Vlatko Vedral. Colloquium: The physics of Maxwell's demon and information. Reviews in Modern Physics, 81:1-23, Jan 2009. DOI: 10.1103/RevModPhys.81.1. URL http://link.aps.org/doi/10.1103/ RevModPhys . 81.1.

[8] Leonard Szilard. Über die Entropieverminderung in einem thermodynamischen System bei Eingriffen intelligenter Wesen. Zeitschrift für Physik, 53(11):840-856, 1929. DOI: 10.1007/BF01341281. URL http://dx.doi. org/10.1007/BF01341281.

[9] Rolf Landauer. Irreversibility and Heat Generation in the Computing Process. IBM Journal of Research and Development, 5(3):183191, July 1961. ISSN 0018-8646. DOI: 10.1147/rd.53.0183.

[10] Charles H. Bennett. The thermodynamics of computation-a review. International Journal of Theoretical Physics, 21(12): 905-940, 1982. ISSN 1572-9575. DOI: 10.1007/BF02084158. URL http://dx.doi . org/10.1007/BF02084158.

[11] Martin B. Plenio and V. Vitelli. The physics of forgetting: Landauer's erasure principle and information theory. Contemporary Physics, 42(1):25-60, 2001. DOI: $10.1080 / 00107510010018916$. URL http://dx.doi.org/10.1080/ 00107510010018916.

[12] Lidia del Rio, Johan Åberg, Renato Renner, Oscar C. O. Dahlsten, and Vlatko Vedral. The thermodynamic meaning of negative entropy. Nature, 474:61-63, 2011. DOI: 10.1038/nature10123. URL http://dx.doi.org/10. 1038/nature10123.

[13] David Reeb and Michael M. Wolf. An improved Landauer principle with finite-size corrections. New Journal of Physics, 16(10):103011, 2014. DOI: 10.1088/1367-2630/16/10/103011. URL http://iopscience.iop.org/article/ 10.1088/1367-2630/16/10/103011.

[14] Claude E. Shannon. A mathematical theory of communication. Bell System Technical Journal, 27(3):379-423, July 1948. ISSN 0005-8580. DOI: $10.1002 / j .1538-7305.1948 . t b 01338 . x$.

[15] Michael A. Nielsen and Isaac L. Chuang. Quantum Computation and Quantum Information. Cambridge: Cambridge University Press, 2000. ISBN 978-0521635035.

[16] Thomas M. Cover and Joy A. Thomas. Elements of Information Theory. John Wiley and Sons, Inc., 2 edition, 2005. ISBN 9780471748823. DOI: 10.1002/047174882X. URL http://dx.doi.org/10.1002/ 047174882X.

[17] Robert Alicki and Mark Fannes. Entanglement boost for extractable work from ensembles of quantum batteries. Physical Review E, 87:042123, Apr 2013. DOI: 10.1103/Phys- 
RevE.87.042123. URL http://link.aps . org/doi/10.1103/PhysRevE.87.042123.

[18] Martí Perarnau-Llobet, Karen V. Hovhannisyan, Marcus Huber, Paul Skrzypczyk, Nicolas Brunner, and Antonio Acín. Extractable Work from Correlations. Physical Review X, 5:041011, Oct 2015. DOI: 10.1103/PhysRevX.5.041011. URL http://link.aps. org/doi/10.1103/PhysRevX.5.041011.

[19] Manabendra Nath Bera, Arnau Riera, Maciej Lewenstein, and Andreas Winter. Generalized laws of thermodynamics in the presence of correlations. Nature Communications, 8:2180, 2017. DOI: doi:10.1038/s41467-017-02370-x. URL https: //www . nature.com/articles/ s41467-017-02370-x.

[20] Anthony J. Short. Equilibration of quantum systems and subsystems. New Journal of Physics, 13(5):053009, 2011. DOI: 10.1088/1367-2630/13/5/053009. URL http://iopscience.iop.org/article/ 10.1088/1367-2630/13/5/053009.

[21] John Goold, Marcus Huber, Arnau Riera, Lidia del Rio, and Paul Skrzypczyk. The role of quantum information in thermodynamics: a topical review. Journal of Physics A: Mathematical and Theoretical, 49(14):143001, 2016. DOI: $10.1088 / 1751-8113 / 49 / 14 / 143001$. URL http://iopscience.iop.org/article/ 10.1088/1751-8113/49/14/143001.

[22] Lídia del Rio, Adrian Hutter, Renato Renner, and Stephanie Wehner. Relative thermalization. Physical Review E, 94:022104, Aug 2016. DOI: 10.1103/PhysRevE.94.022104. URL http://link .aps .org/doi/10.1103/ PhysRevE. 94.022104.

[23] Christian Gogolin and Jens Eisert. Equilibration, thermalisation, and the emergence of statistical mechanics in closed quantum systems. Reports on Progress in Physics, 79(5):056001, $2016 . \quad$ DOI: 10.1088/0034-4885/79/5/056001. URL http://iopscience.iop.org/article/ 10.1088/0034-4885/79/5/056001.

[24] Sandu Popescu, Anthony J. Short, and Andreas Winter. Entanglement and the foundations of statistical mechanics. Nature Physics, 2:745758, 2006. DOI: 10.1038/nphys444. URL http://dx.doi.org/10.1038/nphys444.

[25] Fernando G. S. L. Brandão, Michał Horodecki, Jonathan Oppenheim, Joseph M. Renes, and
Robert W. Spekkens. Resource theory of quantum states out of thermal equilibrium. Physical Review Letters, 111:250404, Dec 2013. DOI: 10.1103/PhysRevLett.111.250404. URL http://link . aps .org/doi/10.1103/ PhysRevLett.111.250404.

[26] Oscar C. O. Dahlsten, Renato Renner, Elisabeth Rieper, and Vlatko Vedral. Inadequacy of von Neumann entropy for characterizing extractable work. New Journal of Physics, 13(5):053015, 2011. DOI: 10.1088/1367-2630/13/5/053015. URL http://iopscience.iop.org/article/ 10.1088/1367-2630/13/5/053015.

[27] Johan Åberg. Truly work-like work extraction via a single-shot analysis. $\mathrm{Na}$ ture Communications, 4:1925, 2013. DOI: 10.1038/ncomms2712. URL http://dx. doi . org/10.1038/ncomms2712.

[28] Michał Horodecki and Jonathan Oppenheim. Fundamental limitations for quantum and nanoscale thermodynamics. Nature Communications, 4:2059, 2013. DOI: $10.1038 /$ ncomms3059. URL http: //dx.doi.org/10.1038/ncomms3059.

[29] Paul Skrzypczyk, Anthony J. Short, and Sandu Popescu. Work extraction and thermodynamics for individual quantum systems. $\mathrm{Na}$ ture Communications, 5:4185, 2014. DOI: 10.1038/ncomms5185. URL http://dx.doi . org/10.1038/ncomms5185.

[30] Fernando G. S. L. Brandao, Michał Horodecki, Nelly Ng, Jonathan Oppenheim, and Stephanie Wehner. The second laws of quantum thermodynamics. Proceedings of the $\mathrm{Na}$ tional Academy of Sciences, 112:3275-3279, 2015. DOI: doi:10.1073/pnas.1411728112. URL http: //www . pnas .org/content/112/ 11/3275.

[31] Piotr Ćwikliński, Michał Studziński, Michał Horodecki, and Jonathan Oppenheim. Limitations on the Evolution of Quantum Coherences: Towards Fully Quantum Second Laws of Thermodynamics. Physical Review Letters, 115:210403, Nov 2015. DOI: 10.1103/PhysRevLett.115.210403. URL http://link.aps.org/doi/10.1103/ PhysRevLett.115.210403.

[32] Matteo Lostaglio, Kamil Korzekwa, David Jennings, and Terry Rudolph. Quantum Coherence, Time-Translation Symmetry, and Ther- 
modynamics. Physical Review X, 5:021001, Apr 2015. DOI: 10.1103/PhysRevX.5.021001. URL http://link.aps.org/doi/10.1103/ PhysRevX.5.021001.

[33] Dario Egloff, Oscar C. O. Dahlsten, Renato Renner, and Vlatko Vedral. A measure of majorization emerging from singleshot statistical mechanics. New Journal of Physics, 17(7):073001, 2015. DOI: 10.1088/1367-2630/17/7/073001. URL http://iopscience.iop.org/article/ 10.1088/1367-2630/17/7/073001.

[34] Matteo Lostaglio, David Jennings, and Terry Rudolph. Description of quantum coherence in thermodynamic processes requires constraints beyond free energy. Nature Communications, 6:6383, 2015. DOI: $10.1038 /$ ncomms7383. URL http: //dx.doi.org/10.1038/ncomms7383.

[35] Manabendra Nath Bera, Antonio Acín, Marek Kuś, Morgan Mitchell, and Maciej Lewenstein. Randomness in quantum mechanics: Philosophy, physics and technology. Reports on Progress in Physics, 80(12):124001, 2017. DOI: $10.1088 / 1361-6633 /$ aa8731. URL http://iopscience.iop.org/article/ 10.1088/1361-6633/aa8731.

[36] Florian Hulpke, Uffe V. Poulsen, Anna Sanpera, Aditi Sen(De), Ujjwal Sen, and Maciej Lewenstein. Unitarity as preservation of entropy and entanglement in quantum systems. Foundations of Physics, 36(4):477-499, 2006. ISSN 1572-9516. DOI: 10.1007/s10701005-9035-7. URL http://dx.doi.org/10. 1007/s10701-005-9035-7.

[37] Christopher Jarzynski. Hamiltonian derivation of a detailed fluctuation theorem. Journal of Statistical Physics, 98(1):77102, Jan 2000. ISSN 1572-9613. DOI: 10.1023/A:1018670721277. URL https: //doi.org/10.1023/A: 1018670721277.

[38] Massimiliano Esposito, Upendra Harbola, and Shaul Mukamel. Nonequilibrium fluctuations, fluctuation theorems, and counting statistics in quantum systems. Rev. Mod. Phys., 81: 1665-1702, Dec 2009. DOI: 10.1103/RevModPhys.81.1665. URL https://link.aps. org/doi/10.1103/RevModPhys.81.1665.

[39] Massimiliano Esposito, Katja Lindenberg, and Christian Van den Broeck. Entropy production as correlation between system and reservoir.
New Journal of Physics, 12(1):013013, 2010. DOI: 10.1088/1367-2630/12/1/013013. URL http://iopscience.iop.org/article/ 10.1088/1367-2630/12/1/013013.

[40] Philipp Strasberg, Gernot Schaller, Tobias Brandes, and Massimiliano Esposito. Quantum and Information Thermodynamics: A Unifying Framework Based on Repeated Interactions. Physical Review X, 7:021003, Apr 2017. DOI: $10.1103 /$ PhysRevX.7.021003. URL https://link.aps.org/doi/10.1103/ PhysRevX.7.021003.

[41] Takahiro Sagawa. Second law-like inequalities with quantum relative entropy: an introduction. Lectures on Quantum Computing, Thermodynamics and Statistical Physics, volume 8. World Scientific, Singapore, 2012. DOI: 10.1142/9789814425193_0003. URL https://doi.org/10.1142/ 9789814425193_0003.

[42] A. Puglisi, A. Sarracino, and A. Vulpiani. Temperature in and out of equilibrium: A review of concepts, tools and attempts. Physics Reports, 709-710:1-60, 2017. ISSN 0370-1573. DOI: https://doi.org/10.1016/j.physrep.2017.09.001.

URL https://www.sciencedirect.com/ journal/physics-reports/vol/709/ suppl/C.

[43] Carlo Sparaciari, Jonathan Oppenheim, and Tobias Fritz. Resource theory for work and heat. Physical Review A, 96:052112, Nov 2017. DOI: $10.1103 /$ PhysRevA.96.052112. URL https://link.aps.org/doi/10.1103/ PhysRevA.96.052112.

[44] Henrik Wilming, Rodrigo Gallego, and Jens Eisert. Axiomatic characterization of the quantum relative entropy and free energy. Entropy, 19(6), 2017. ISSN 1099-4300. DOI: 10.3390/e19060241. URL http: //www . mdpi.com/1099-4300/19/6/241.

[45] Markus P. Müller. Correlating thermal machines and the second law at the nanoscale. arXiv[quant-ph]:1707.03451, 2017. URL https://arxiv.org/abs/1707.03451.

[46] Edwin T. Jaynes. Information Theory and Statistical Mechanics. Physical Review, 106: 620-630, May 1957. DOI: 10.1103/PhysRev.106.620. URL https://link.aps .org/ doi/10.1103/PhysRev.106.620.

[47] Edwin T. Jaynes. Information Theory and Statistical Mechanics. II. Physical Review, 
108:171-190, Oct 1957. DOI: 10.1103/PhysRev.108.171. URL https://link.aps.org/ doi/10.1103/PhysRev.108.171.

[48] Wiesław Pusz and Stanisław L. Woronowicz. Passive states and KMS states for general quantum systems. Communications in Mathematical Physics, 58(3):273-290, 1978. ISSN 14320916. DOI: 10.1007/BF01614224. URL http: //dx.doi.org/10.1007/BF01614224.

[49] Andrew Lenard. Thermodynamical proof of the Gibbs formula for elementary quantum systems. Journal of Statistical Physics, 19(6): 575-586, 1978. ISSN 1572-9613. DOI: 10.1007/BF01011769. URL http://dx.doi. org/10.1007/BF01011769.

[50] Lluís Masanes and Jonathan Oppenheim. A general derivation and quantification of the third law of thermodynamics. $\mathrm{Na}$ ture Communications, 8:14538, 2017. DOI: 10.1038/ncomms14538. URL http://dx. doi.org/10.1038/ncomms14538.

[51] Nicole Yunger Halpern and Joseph M. Renes. Beyond heat baths: Generalized resource theories for small-scale thermodynamics. Physical Review E, 93:022126, Feb 2016. DOI: 10.1103/PhysRevE.93.022126. URL https://link.aps.org/doi/10.1103/ PhysRevE.93.022126.

[52] Nicole Yunger Halpern. Beyond heat baths II: Framework for generalized thermodynamic resource theories. Journal of Physics A: Mathematical and Theoretical, 51:094001, 2018. DOI: 10.1088/1751-8121/aaa62f. URL http://iopscience.iop.org/article/ 10.1088/1751-8121/aaa62f/meta.

[53] Matteo Lostaglio, David Jennings, and Terry Rudolph. Thermodynamic resource theories, non-commutativity and maximum entropy principles. New Journal of Physics, 19(4):043008, 2017. DOI: 10.1088/1367-2630/aa617f. URL http://iopscience.iop.org/article/ 10.1088/1367-2630/aa617f.

[54] Yelena Guryanova, Sandu Popescu, Anthony J. Short, Ralph Silva, and Paul Skrzypczyk. Thermodynamics of quantum systems with multiple conserved quantities. Nature Communications, 7:12049, 2016. DOI: $10.1038 /$ ncomms12049. URL http: //dx.doi.org/10.1038/ncomms12049.

[55] Nicole Yunger Halpern, Philippe Faist, Jonathan Oppenheim, and Andreas Win- ter. Microcanonical and resource-theoretic derivations of the thermal state of a quantum system with noncommuting charges. Nature Communications, 7:12051, 2016. DOI: $10.1038 /$ ncomms12051. URL http: //dx.doi.org/10.1038/ncomms12051.

[56] Zahra B. Khanian, Manabendra Nath Bera, Arnau Riera, Maciej Lewenstein, and Andreas Winter. Resource theory of work and heat and everything else: basing thermodynamics of multiple non-commuting conserved quantities on an asymptotic equivalence principle. In preparation, 2018. 


\section{A Alternative formulation of the Clausius statement for the second law}

Lemma 22 (Clausius statement). No isoentropic equilibration process is possible whose sole result is the transfer of bound energy (i.e. heat) from an equilibrium state with inverse temperature $\beta_{C}$ to another equilibrium state with inverse temperature $\beta_{H}<\beta_{C}$.

Proof. In order to prove it, we need to show that the iso-entropic equilibration process cannot lead to a negative $\Delta \beta=\beta_{H}-\beta_{C}$. Further, that any iso-entropic process that leads to such increase must require a supply of energy. First consider the case where $\beta_{C}=\beta_{H}(\Delta \beta=0)$, which means the corresponding states $\gamma_{C}$ and $\gamma_{H}$ are in equilibrium between each other and jointly in the min-energy state. The min-energy principle says that any iso-entropic transformation to make $\beta_{C} \neq \beta_{H}$ is bound to increase the sum of their individual min-energy. Therefore it requires an inflow of energy, which is nothing but introduction of work.

Note that for a completely passive state $\gamma$ with given Hamiltonian $H$ and $\beta$, the change in bound energy, which is nothing but heat in our definition, due to an infinitesimal change in entropy $d S(\gamma)$, is given by

$$
d E(\gamma)=\frac{1}{\beta} d S(\gamma)
$$

Therefore the larger $\beta$, the smaller the change in internal energy for a fixed infinitesimal change in entropy of the state. Now assume by contradiction that $\beta_{C}>\beta_{H}$. Any flow of heat from $\gamma_{C}$ to $\gamma_{H}$ will lead to a reduction of entropy in the former. That will also lead to an equal increase of of the same in the latter. A very small amount of bound energy will result in a infinitesimal entropy flow, say $d S$ from $\gamma_{C}$ to $\gamma_{H}$. However the change in internal energy $\left(d E\left(\gamma_{C / H}\right)=\frac{d S}{\beta_{C / H}}\right)$ would not be equal and that is $-d E\left(\gamma_{C}\right)<d E\left(\gamma_{H}\right)$, for $\beta_{C}>\beta_{H}$. As a consequence, in this iso-entropic process the overall energy is bound to increase, which is not possible without, again, an influx of energy or work. Therefore, without external work the process will never take place spontaneously.

\section{B Alternative formulation of the Kelvin-Planck statement for the second law}

Lemma 23 (Kelvin-Planck statement). No isoentropic equilibration process is possible whose sole result is the absorption of bound energy (heat) from an equilibrium state and its complete conversion into work.

Proof. To prove the Kelvin-Planck statement, we consider the following two completely passive states $\gamma_{A}$ and $\gamma_{B}$ with inverse temperatures $\beta_{A}$ and $\beta_{B}$, respectively, which undergo an isoentropic equilibration transformation as

$$
\gamma_{A} \otimes \gamma_{B} \otimes|0\rangle\left\langle\left. 0\right|_{W} \stackrel{\Lambda_{A B W}^{E P}}{\longrightarrow} \gamma_{A B} \otimes \mid W\right\rangle\left\langle\left. W\right|_{W},\right.
$$

where $\gamma_{A B}$ is the final joint equilibrium state with inverse temperature $\beta_{A B}$. Since the initial states are completely passive states, a non-zero $W$ can only result from heat transfer. Let us say $\beta_{A}<\beta_{B}$, thus $\beta_{A}<\beta_{A B}<\beta_{B}$. In this case the heat flows out from $A$, say by an amount $\Delta Q_{A}$, with an associated decrease in its entropy. Again the information conservation of whole process guarantees an entropy increase in $B$. Therefore, there has to be an associated increase in bound energy (heat) content in $\gamma_{B}$. As a result, a part of $\Delta Q_{A}$ could be converted to work and that is

$$
W \leqslant \Delta Q_{A}-\Delta Q_{B}
$$

Note that any transfer of heat, i.e. $-\Delta Q_{A}>0$, also bounds $\Delta Q_{B}>0$. As a consequence, heat cannot be converted into work completely. 